OPEN ACCESS

Edited by:

Yuji Morita,

Aichi Gakuin University, Japan

Reviewed by:

Ashok K. Chopra,

University of Texas Medical Branch,

USA

Juan M. Tomas,

University of Barcelona, Spain

Morgan Beeby

Imperial College London, UK

*Correspondence:

Jonathan G. Shaw

j.g.shaw@sheffield.ac.uk

Specialty section:

This article was submitted to Infectious Diseases,

a section of the journal

Frontiers in Microbiology

Received: 30 June 2016

Accepted: 29 August 2016 Published: 07 September 2016

Citation:

Zhao Y-H and Shaw JG (2016) Cross-Talk between the Aeromonas hydrophila Type III Secretion System

and Lateral Flagella System.

Front. Microbiol. 7:1434.

doi: 10.3389/fmicb.2016.01434

\section{Cross-Talk between the Aeromonas hydrophila Type III Secretion System and Lateral Flagella System}

\author{
Yu-Hang Zhao and Jonathan G. Shaw* \\ Department of Infection, Immunity and Cardiovascular Disease, University of Sheffield, Sheffield, UK
}

Aeromonas hydrophila is responsible for aeromonad septicaemia in fish, and gastroenteritis and wound infections in humans. The type III secretion system (T3SS) is utilized by aeromonads to inject protein effectors directly into host cells. One of the major genetic regulators of the T3SS in several bacterial species is the AraC-like protein ExsA. Previous studies have suggested a link between T3SS regulation and lateral flagella expression. The aim of this study was to determine the genetic regulation of the T3SS and its potential interaction with the lateral flagella system in A. hydrophila. To investigate the genes encoding the T3SS regulatory components exSA, exsD, exsC, and exsE were mutated and the activities of the T3SS promoters were measured in wild type and mutant backgrounds demonstrating a regulatory network. The Exs proteins were shown to interact with each other by BACTH assay and Far-Western Blot. The findings suggested a regulatory cascade in which ExsE was bound to the chaperone protein ExsC. When ExsC was free it sequestered the anti-activator ExsD thus stopping the inhibition of the T3SS master regulator ExsA allowing T3SS expression. The T3SS regulatory components were also shown to affect the expression of the lateral flagella system. The activities of the lateral flagella promoters were shown to be repressed by the absence of ExsD and ExsE, suggesting that the T3SS master regulator ExsA was a negative regulator of the lateral flagella system.

Keywords: Aeromonas, swarming, motility, lateral flagella, T3SS

\section{INTRODUCTION}

Aeromonas species are ubiquitous water-borne Gram-negative bacteria that are able to cause a variety of diseases in poikilothermic animals and humans. The psychrophilic Aeromonas species represented by A. salmonicida predominantly cause furunculosis in salmonid fish as well as many other fish species (McCarthy and Rawle, 1975; Cipriano and Austin, 2011). Human infections are caused by mesophilic aeromonads, of which A. hydrophila, A. caviae, and A. veronii biovar sobria account for $85 \%$ of clinical isolates (Parker and Shaw, 2011). Human infections by aeromonads often result in gastrointestinal disease and wound infection, while septicaemia less commonly occurs in the immunocompromised host (Janda and Abbott, 2010).

The pathogenicity of Aeromonas species is associated with a variety of virulence factors such as lipopolysaccharide, S-Layer, enterotoxins, the polar and lateral flagella systems as well as secretion systems (Lowry et al., 2014). The colonization approaches that involve the presence of flagella systems are swimming and swarming (Harshey, 2003). Aeromonads swim using a single polar 
flagellum, but approximately $60 \%$ of motile Aeromonas strains possess a second lateral flagella system for swarming motility (Gavin et al., 2002; Kirov et al., 2002). The lateral flagella system in A. hydrophila was also found to be involved in host cell adherence and biofilm formation (Gavin et al., 2002; Kirov, 2003).

In A. hydrophila $\mathrm{AH}-3,38$ genes of the lateral flagella system are categorized into nine putative operons, expression of which is controlled in a hierarchal fashion (Wilhelms et al., 2013).

Evidence in A. hydrophila AH-1 has shown that the expression level of the two tandem lateral flagellin genes lafA 1 and lafA2 are significantly reduced in exs $D$ and aopN mutants, genes that are associated with the Type III secretion system (T3SS). However, $\Delta \operatorname{exs} A \Delta \operatorname{exs} D$ and the $\Delta \operatorname{exs} A \Delta \operatorname{aop} N$ double mutations restored the secretion level of the LafA flagellin protein (Yu et al., 2007). This finding indicated potential cross-talk between the lateral flagella system and the T3SS in A. hydrophila.

Type III secretion systems (T3SS) have been reported in more than 25 species of Gram-negative bacteria and they are able to inject protein effectors across the plasma membrane directly into the host cell cytosol or to secrete pore-forming translocators that helps effector proteins to get through (Cornelis and Van Gijsegem, 2000; Abby and Rocha, 2012). In Aeromonas the T3SS is similar to those reported in Pseudomonas aeruginosa and Yersinia spp. (Vilches et al., 2004).

The assembly of the T3SS requires a high level of energy and resources; thus it is tightly regulated. In $P$. aeruginosa the master regulator of the T3SS is the AraC family protein ExsA. Transcriptional activation of exs $A$ was required for the expression of the T3SS secretion apparatus, translocation machinery and secreted effectors (Hovey and Frank, 1995; Yahr et al., 1995). Moreover, ExsA in A. hydrophila shares $76 \%$ amino acid homology with ExsA in P. aeruginosa (Frank and Iglewski, 1991; Yahr and Frank, 1994; Vilches et al., 2009). As the role of ExsA in $P$. aeruginosa is defined as a transcriptional activator by facilitating RNAP- $\sigma^{70}$ binding onto the T3SS promoter regions, the role of ExsA in A. hydrophila is proposed to be similar.

In $P$. aeruginosa the T3SS master regulator ExsA is controlled by a regulatory cascade of proteins ExsD, ExsC, and ExsE (McCaw et al., 2002; Dasgupta et al., 2004; Rietsch et al., 2005; Urbanowski et al., 2005; Zheng et al., 2007). ExsD is reported to be an anti-activator protein that negatively regulates the T3SS master regulator ExsA through direct protein-protein interaction (McCaw et al., 2002). ExsC is an anti-anti-activator protein that binds to ExsD and prevents it from inhibiting ExsA (Dasgupta et al., 2004). ExsC is the chaperone protein for the secreted protein ExsE, which is found to be a negative regulator of the T3SS (Rietsch et al., 2005). ExsE negatively regulates the T3SS via its physical interactions with ExsC, preventing ExsC from binding to ExsD, thus allowing ExsD to be free to bind and inhibit the transcriptional activation from ExsA (Urbanowski et al., 2005).

In comparison with $P$. aeruginosa, the regulation of the T3SS has not been intensively studied in A. hydrophila. Vilches et al. (2004), the complete T3SS region of $A$. hydrophila was sequenced, but the regulation of the T3SS in A. hydrophila still largely unknown. The ExsA and ExsD proteins in A. hydrophila SSU strain had similar effects on the regulation of the T3SS to those found in $P$. aeruginosa using mutagenesis and over-expression assays (Sha et al., 2007). In A. hydrophila AH-3, quantitative RTPCR results for aopN and aexT mRNA production showed 60$85 \%$ decrease in a $\triangle$ exs $A$ mutant background and the promoters activities aopN and aexT genes were also significantly decreased (Vilches et al., 2009). All these findings suggest that ExsA, like its homologue in $P$. aeruginosa, is likely to be the master regulator of the T3SS in A. hydrophila. However, there is little evidence of ExsC and ExsE involvement in the regulation of the T3SS in Aeromonas species. In this study we demonstrate the presence of a T3SS regulatory cascade in A. hydrophila and provide further evidence for the cross-talk between the T3SS regulators and expression of the lateral flagella system.

\section{MATERIALS AND METHODS}

\section{Media, Bacterial Strains, Plasmids, Primers and Antibiotics}

The bacterial strains used in this study are listed in Table $\mathbf{1}$. Plasmids used in this study are listed in Table 2. Escherichia coli strains were grown on Luria Bertani (LB) Miller agar and in LB Miller broth, while A. hydrophila strains were grown on Columbia Blood Agar (Oxoid, Basingstoke, UK) and in LB Miller broth. A. hydrophila strains were incubated at $30^{\circ} \mathrm{C}$ while E. coli strains were incubated at $37^{\circ} \mathrm{C}$. Kanamycin $[\mathrm{Km}](50 \mu \mathrm{g} / \mathrm{ml})$, streptomycin [Sm] $(50 \mu \mathrm{g} / \mathrm{ml})$, ampicillin [Amp] $(100 \mu \mathrm{g} / \mathrm{ml})$, rifampicin [Rif] $(50 \mu \mathrm{g} / \mathrm{ml})$, chloramphenicol [Cm] $(50 \mu \mathrm{g} / \mathrm{ml})$ and gentamycin $[\mathrm{Gm}](50 \mu \mathrm{g} / \mathrm{ml})$ were added when required. Primers used in this study are listed in Supplementary Table S1.

\section{DNA Techniques}

General DNA manipulation methods involving DNA restriction endonuclease, T4 DNA ligase and alkaline phosphatase were used as recommended by the supplier (New England Biolabs) to construct plasmids in this study, including pKNG101 constructs, pET28a constructs, pMAL-c5x constructs, pKAGb-2(-) constructs, pKT25 constructs, pKNT25 constructs, pUT18 constructs and pUT18C constructs. PCR amplification was performed using Platinum Pfx DNA polymerase (Invitrogen) while PCR screening was performed using Taq DNA polymerase (New England Biolabs).

\section{Mini-Tn5Km1 Mutagenesis}

Conjugal transfer of pUT-mini-Tn5Km from E. coli S17-1 $\lambda$ pir to A. hydrophila $\mathrm{AH}-3 \mathrm{R}$ was performed using a filter mating technique. Bacterial conjugation was allowed to proceed for $6-8 \mathrm{~h}$ at $30^{\circ} \mathrm{C}$ on sterile nitrocellulose filters $(0.45 \mathrm{~mm}$ pore size) placed onto a Blood agar plate. Serial dilutions of the mating mix were plated on LB agar supplemented with Rif and $\mathrm{Km}$, the latter added in order to select for mini-Tn5Km. All mutants were then analyzed for the presence of the transposon by Southern hybridisations of PstI chromosomal DNA digestions, as described before (Gryllos et al., 2001). As no PstI restriction sites were present in the transposon, variable size bands larger than the transposon were observed for each mutant. To determine the site 
TABLE 1 | Bacterial strains used in this study.

\begin{tabular}{|c|c|c|}
\hline Name of strains & Description & Reference \\
\hline \multicolumn{3}{|l|}{ Aeromonas hydrophila strains } \\
\hline $\mathrm{AH} 3 \mathrm{R}$ & Wild type Aeromonas hydrophila AH3 strain, Rif ${ }^{R}$ & $\begin{array}{l}\text { Dr. J. Tomás University of } \\
\text { Barcelona }\end{array}$ \\
\hline A5 & AHR strain with miniTn5Km insertion in exsD, $\mathrm{Km}^{\mathrm{R}}$ & This study \\
\hline A25 & AHR strain with miniTn5 $\mathrm{Km}$ insertion in exs $D, \mathrm{Km}^{\mathrm{R}}$ & This study \\
\hline exsA mutant & AH3R strain with exsA knocked out by the insertion of $\mathrm{Km}^{\mathrm{R}}$ cassette & This study \\
\hline exsC mutant & AH3R strain with exs $C$ knocked out by the insertion of $\mathrm{Km}^{R}$ cassette & This study \\
\hline exs $D$ mutant & AH3R strain with exs $D$ knocked out by the insertion of $\mathrm{Km}^{\mathrm{R}}$ cassette & This study \\
\hline exsE mutant & $\mathrm{AH} 3 \mathrm{R}$ strain with exsE knocked out by the insertion of $\mathrm{Km}^{\mathrm{R}}$ cassette & This study \\
\hline lafk mutant & AH3R strain with lafK knocked out by the insertion of $\mathrm{Km}^{\mathrm{R}}$ & This study \\
\hline AH3R-PascN & Reporter plasmid pKAG-PascN conjugated into AH3R wild type & This study \\
\hline AH3R-PexsA & Reporter plasmid pKAG-PexsA conjugated into AH3R wild type & This study \\
\hline AH3R-PexsD & Reporter plasmid pKAG-PexsD conjugated into AH3R wild type & This study \\
\hline AH3R-PfliM & Reporter plasmid pKAG-PfliM conjugated into AH3R wild type & This study \\
\hline AH3R-PlafK & Reporter plasmid pKAG-PlafK conjugated into AH3R wild type & This study \\
\hline AH3R-PflgM & Reporter plasmid pKAG-PflgM conjugated into AH3R wild type & This study \\
\hline AH3R-PflgA & Reporter plasmid pKAG-PflgA conjugated into AH3R wild type & This study \\
\hline AH3R-PflgB & Reporter plasmid pKAG-PflgB conjugated into AH3R wild type & This study \\
\hline AH3R-Pmaf & Reporter plasmid pKAG-Pmaf conjugated into AH3R wild type & This study \\
\hline AH3R-PlafA & Reporter plasmid pKAG-PlafA conjugated into AH3R wild type & This study \\
\hline AH3R-PlafB & Reporter plasmid pKAG-PlafB conjugated into AH3R wild type & This study \\
\hline AH3R-PlafX & Reporter plasmid pKAG-PlafX conjugated into AH3R wild type & This study \\
\hline \multicolumn{3}{|l|}{ Escherichia coli strains } \\
\hline$D H 5 \alpha$ & $\begin{array}{l}\mathrm{F}-, \text { } 980 \text { lacZ } \Delta \mathrm{M} 15, \Delta(\text { lacZYA-argF), U169, recA1, endA1, hsdR17, (rK-, mK+), phoA, } \\
\text { supE44, } \lambda \text { - thi-1, gyrA96, relA1 }\end{array}$ & Hanahan, 1983 \\
\hline CC118- $\lambda$ pir & phoA20, thi-1, rspE, rpoB, argE, (Am), recA1, phage $\lambda$ pir & Herrero et al., 1990 \\
\hline S17-1- $\lambda$ pir & $\mathrm{Sm}^{\mathrm{R}}, \mathrm{F}-, \mathrm{rec} A$, hsdR, RP4-2 (Tc::Mu) (Km::Tn7), phage $\lambda$ pir & Miller, 1972 \\
\hline Sm10- $\lambda$ pir & KmR, thi-1, thr, leu, tonA, lacY, supE, recA::RP4-2-Tc::Mu, phage $\lambda$ pir & Miller and Mekalanos, 1988 \\
\hline HB101 & 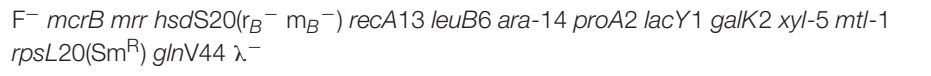 & Hanahan, 1983 \\
\hline BL21(DE3) & $\mathrm{F}^{-}$ompT gal dcm lon hsdS $S_{B}\left(r_{B}^{-} m_{B}^{-}\right) \lambda(\mathrm{DE} 3)$ & Novagen \\
\hline C41(DE3) & ompT hsdSB (rB- mB-) gal dcm (DE3) & Lucigen \\
\hline BL21Star ${ }^{T M}(D E 3)$ & $\mathrm{F}^{-}$omp $\mathrm{T} h \mathrm{sd} \mathrm{S}_{B}\left(\mathrm{r}_{B}^{-}, \mathrm{m}_{B}^{-}\right) \mathrm{gal}$ dcm rne131 (DE3) & Invitrogen \\
\hline BTH101 & $\mathrm{F}^{-}$, cya-99, araD139, galE15, galK16, rpsL1(Str $\left.{ }^{\mathrm{R}}\right)$, hsdR2, mcrA1, mcrB1 & Euromedex \\
\hline E. coli ER2523 (NEB Express) & $\begin{array}{l}\text { fhuA2 [lon] ompT gal sulA11 R(mcr-73::miniTn10-TetS)2 [dcm] R(zgb-210::Tn10-TetS) } \\
\text { endA1 } \Delta \text { (mcrC-mrr)114::IS10 }\end{array}$ & New England BioLabs \\
\hline
\end{tabular}

of the transposon insertion, chromosomal DNA was isolated and direct genomic sequencing was employed using 24-mer primers were designed to the $\mathrm{O}$ and the I end of transposon Tn5 and a 99 cycle polymerase sequencing reaction

\section{Nucleotide Sequencing and Sequence Analysis}

Double-stranded DNA sequencing was performed by using the Sanger dideoxy-chain termination method with the Abi Prism dye terminator cycle sequencing kit (Perkin Elmer). DNA fragments were ligated into appropriate primers and sequenced using an ABI PRISM 377 DNA sequencer (PerkinElmer Corporation). Universal or custom designed primers were employed in sequencing the ends of the DNA inserts (Table 3). Following the first sequencing reaction and whenever required, primers were designed until the inserts' sequences were complete. Primers used for DNA sequencing were purchased from Eurogentec. For chromosomal walking to extend the sequence into flanking regions direct genomic sequencing was used. Custom 24-mer primers were designed to a known nucleotide sequence of the $\mathrm{O}$ and the I end of trasposon Tn5 and were used with sheared $A$. hydrophila genomic DNA in a 99 cycle polymerase reaction using the BigDye terminator mix according to the manufacturer's instructions (P.E.-Applied Biosystems).

\section{Construction of Defined Insertional exs $A$, exsC and lafK Mutants}

In order to facilitate a knockout in the exs $A$, exs $C$ or lafK genes, a selectable marker was inserted in the middle of the gene. Mutants were created by the insertion of the Tn5 derived kanamycin resistance cartridge ( $n p t I I)$ from pUC4-KIXX (Amersham). This cartridge contains an outward reading promoter that drives the transcription of downstream genes when inserted in the correct orientation. Although the cartridge insertions ensure transcription of downstream genes, the regulation and expression 
TABLE 2 | Plasmids used in this study.

\begin{tabular}{|c|c|c|}
\hline Name of plasmids & Characteristics & Reference \\
\hline pBBR1MCS & Broad-host-range plasmid, $\mathrm{Cm}^{R}$ & Kovach et al., 1994 \\
\hline pBBR1MCS-5 & Broad-host-range plasmid, $\mathrm{Gm}^{\mathrm{R}}$ & Kovach et al., 1994 \\
\hline pBBexsCEB & exsCEB region cloned into pBBR1MCS & This study \\
\hline pBBexsCKm $m^{R}$ & exsC::Km $\mathrm{Km}^{R}$ nockout ligated with pBBR1MCS & This study \\
\hline pBBR5exsA & exsA ligated with pBBR1MCS-5 & This study \\
\hline pUTmini-Tn5Km1 & Suicide vector carrying $\mathrm{Tn} 5, \mathrm{Amp}^{\mathrm{R}} \mathrm{Km}^{\mathrm{R}}$ & de Lorenzo et al., 1990 \\
\hline pUC4KIXX & Source of $\mathrm{Km}^{\mathrm{R}}$ cassette (nptll) & Amersham Pharmacia \\
\hline pJMK30 & pUC19 derivative, containing a $\mathrm{Km}^{\mathrm{R}}$ cassette (AphA-3) from Campylobacter coli & van Vliet et al., 1998 \\
\hline pKNG101 & RK6 derived suicide plasmid, $\mathrm{SacB}, \mathrm{Sm}^{\mathrm{R}}$ & Kaniga et al., 1991 \\
\hline pKNGexsAKm R & exsA:: $\mathrm{Km}^{R}$ knockout ligated with pKNG101 suicide plasmid & This study \\
\hline pKNGexsCKm & exsC::Km $\mathrm{K}^{\mathrm{R}}$ knockout ligated with pKNG101 suicide plasmid & This study \\
\hline pKNGexsDKm ${ }^{R}$ & exsD::Km $\mathrm{K}^{\mathrm{R}}$ knockout ligated with pKNG101 suicide plasmid & This study \\
\hline pKNGexsEKm ${ }^{R}$ & exsE::Km $\mathrm{K}^{\mathrm{R}}$ knockout ligated with pKNG101 suicide plasmid & This study \\
\hline pGEM-3Zf(+) & Cloning vector, used for ISA-cloning in this study, $\mathrm{Amp}^{\mathrm{R}}$ & Promega \\
\hline pET28a & Over-expression vector, T7 promoter, $\mathrm{N}$-terminal His tag, $\mathrm{Km}^{\mathrm{R}}$ & Novagen \\
\hline pET28exsA & exsA ligated in frame with His-tag in pET28a & This study \\
\hline pET28exsC & exsC ligated in frame with His-tag in pET28a & This study \\
\hline pET28exsD & exs $D$ ligated in frame with His-tag in pET28a & This study \\
\hline pET28exsE & exsE ligated in frame with His-tag in pET28a & This study \\
\hline pMAL-c5x & Over-expression vector, malE-, MBP5 coding, $\mathrm{Amp}^{\mathrm{R}}$ & New England BioLabs \\
\hline pMALexsA & exsA ligated in frame with MBP5 gene in pMAL-c5x & This study \\
\hline pMALexsC & exsC ligated in frame with MBP5 gene in PMAL-c5x & This study \\
\hline pMALexsD & exs $D$ ligated in frame with MBP5 gene in PMAL-c5x & This study \\
\hline pMALexsE & exsE ligated in frame with MBP5 gene in PMAL-c5x & This study \\
\hline pKAGb-2(-) & Broad-host-range reporter vector, ori ${ }_{1600}$ carrying promoter-less lac $Z$ gene, $\mathrm{Cm}^{\mathrm{R}}$ & Tabei et al., 2009 \\
\hline pKAG-PascN & Promoter upstream of ascN cloned into pKAG-b2(-) & This study \\
\hline pKAG-PaopN & Promoter upstream of aopN cloned into pKAG-b2(-) & This study \\
\hline pKAG-PexsC & Promoter upstream of exsC cloned into pKAG-b2(-) & This study \\
\hline pKAG-PexsA & Promoter upstream of exsA cloned into pKAG-b2(-) & This study \\
\hline pKAG-PexsD & Promoter upstream of exsD cloned into pKAG-b2(-) & This study \\
\hline pKAG-PfliM & Promoter upstream of fliM cloned into pKAG-b2(-) & This study \\
\hline pKAG-PflgB & Promoter upstream of flgB cloned into pKAG-b2(-) & This study \\
\hline pKAG-PlafA & Promoter upstream of lafA cloned into pKAG-b2(-) & This study \\
\hline pKT25 & pSU40 derivative, lac promoter, allow in-frame fusion at C-terminal of $\mathrm{T} 25, \mathrm{Km}^{\mathrm{R}}$ & Euromedex \\
\hline pKNT25 & pSU40 derivative, lac promoter, allow in-frame fusion at $\mathrm{N}$-terminal of $\mathrm{T} 25, \mathrm{Km}^{\mathrm{R}}$ & Euromedex \\
\hline pUT18 & pUC19 derivative, lac promoter, allow in-frame fusion at $\mathrm{N}$-terminal of $\mathrm{T} 18, \mathrm{Amp}^{\mathrm{R}}$ & Euromedex \\
\hline pUT18C & pUC19 derivative, lac promoter, allow in-frame fusion at C-terminal of $\mathrm{T} 18, \mathrm{Amp}^{\mathrm{R}}$ & Euromedex \\
\hline pKT25-zip & pKT25 derivative, leucine zipper of GCN4 fused at C-terminal of T25, $\mathrm{Km}^{\mathrm{R}}$ & Euromedex \\
\hline pUT18C-zip & pUT18C derivative, leucine zipper of GCN4 fused at C-terminal of T25, $\mathrm{Km}^{R}$ & Euromedex \\
\hline pKT25-exsA & exsA cloned in-frame with pKT25 & This study \\
\hline pKT25-exsC & exs $C$ cloned in-frame with pKT25 & This study \\
\hline pKT25-exsD & exs D cloned in-frame with pKT25 & This study \\
\hline pKT25-exsE & exsE cloned in-frame with pKT25 & This study \\
\hline pKNT25-exsA & exsA cloned in-frame with pKNT25 & This study \\
\hline pKNT25-exsC & exsC cloned in-frame with pKNT25 & This study \\
\hline pKNT25-exs D & exs $D$ cloned in-frame with pKNT25 & This study \\
\hline pKNT25-exsE & exsE cloned in-frame with pKNT25 & This study \\
\hline pUT18-exsA & exsA cloned in-frame with pUT18 & This study \\
\hline pUT18-exsC & exs $C$ cloned in-frame with pUT18 & This study \\
\hline pUT18-exsD & exs $D$ cloned in-frame with pUT18 & This study \\
\hline pUT18-exsE & exsE cloned in-frame with pUT18 & This study \\
\hline
\end{tabular}


TABLE 2 | Continued

\begin{tabular}{|c|c|c|}
\hline Name of plasmids & Characteristics & Reference \\
\hline pUT18C-exsA & exsA cloned in-frame with pUT18C & This study \\
\hline pUT18C-exsC & exsC cloned in-frame with pUT18C & This study \\
\hline pUT18C-exsD & exs $D$ cloned in-frame with pUT18C & This study \\
\hline pUT18C-exsE & exsE cloned in-frame with pUT18C & This study \\
\hline
\end{tabular}

levels of these genes could be altered. For each mutant the $1.4 \mathrm{~kb}$ SmaI digested kanamycin resistance cartridge was inserted into a convenient site within the middle of the gene. If a convenient site was not present, one was created by PCR. Constructs containing the mutated genes were ligated into the suicide vector pKNG101 and transferred into Aeromonas by conjugation to allow allelic exchange as described before (Tabei et al., 2009). After conjugation, a double crossover event was selected by picking colonies that were streptomycin sensitive and kanamycin resistant. The potential mutants were checked by PCR screening using the appropriate amplification primers followed by sequencing (provided by Core Genomic Facility, University of Sheffield).

\section{Construction of exsD and exsE Mutant Using Isothermal Assembly (ISA)}

Isothermal assembly was used to assemble multiple DNA fragments into one plasmid vector in a single reaction (Gibson et al., 2009). ISA required three DNA fragments and one digested plasmid for the assembly, in which exs $D$ was amplified by PCR into two fragments using primers exs $D$ F1 forward/reverse and exsD F2 forward/reverse (Table 3), each containing adapter sequence overlapping with the plasmid or the kanamycin cassette. The kanamycin cassette was amplified by PCR from plasmid pJMK30 using Pfx DNA polymerase and primers Kan forward/reverse (Table 3), while the plasmid pGEM-3Zf(+) was digested with the restriction enzyme HincII. Each fragment assembled in this reaction should have an approximately $30 \mathrm{bp}$ adapter sequence overlapping each other. Then the fragments, which were equi-molar when added, were mixed together with ISA Buffer (Gibson et al., 2009) and three enzymes: T5 exonuclease, Phusion polymerase and Taq ligase. This mixture was incubated at $50^{\circ} \mathrm{C}$ overnight and transformed into E. coli $\mathrm{DH} 5 \alpha$ to select for ampicillin and kanamycin resistance, generating pGEMexsDKm ${ }^{\mathrm{R}}$. The pGEMexsEKm ${ }^{\mathrm{R}}$ plasmid construct was assembled using the same method. Both constructs were checked by nucleotide sequencing. The exsD::Km ${ }^{\mathrm{R}}$ fragment was then amplified from the pGEMexsDKm ${ }^{\mathrm{R}}$ by PCR using Pfx DNA polymerase with exsD_pGEM forward and exsD_pGEM reverse primers (Table 3). A similar PCR amplification was carried out to obtain the exsE::Km ${ }^{\mathrm{R}}$ fragment (exsE_pGEM forward and exsE_pGEM reverse primers) on the plasmid construct pGEMexsEKm ${ }^{\mathrm{R}}$.

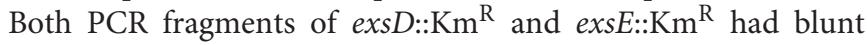
ends and were ligated into SmaI-digested suicide plasmid pKNG101. After ligation, the samples were transformed into competent cells of $E$. coli CC118- $\lambda$ pir to select for kanamycin and

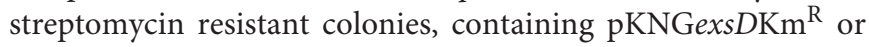

pKNGexsEKm ${ }^{\mathrm{R}}$ constructs. Once the suicide plasmid constructs were prepared, they were transformed into the $E$. coli donor cell S17-1- $\lambda$ pir and then conjugated into A. hydrophila AH3R to allow allelic exchange. A double crossover event was selected by picking up colonies that were streptomycin sensitive and kanamycin resistant. The chromosomal DNA of these potential exs $D$ or exsE mutants were then extracted and used as templates for PCR screening using Taq DNA polymerase with exsD F1 forward and exsD F2 reverse primers, followed by sequencing to confirm the knockout. The same PCR screening and sequencing were carried out on potential exsE mutant using exsE F1 forward and exsE F2 reverse primers.

\section{Swarming Assay}

Swarming motility of $A$. hydrophila strains were assessed on swarming agar plates $(0.5 \% \mathrm{NaCl}, 0.6 \%$ Difco Nutrient Broth, and $0.6 \%$ Eiken agar). A $1 \mathrm{ml}$ volume of the bacterial strains that were pre-cultured in broth media were centrifuged at $15,000 \times g$ for $1 \mathrm{~min}$. A sterile toothpick was used to transfer a small amount (less than a colony size) of the pellet into the center of swarming agar plates. The plates were incubated face-up at $30^{\circ} \mathrm{C}$ overnight. Swarming motility was measured by examining the migration of bacteria across the agar (swarming diameters) from the center toward the periphery of the plate (Kirov et al., 2004). Bacterial cells required for lateral flagella transcriptional fusion assays were grown at $30^{\circ} \mathrm{C}$ overnight and the edge of the bacterial swarm was resuspended in sterile $\mathrm{PBS}$ for the $\beta$-galactosidase assay.

\section{$\beta$-Galactosidase Assay}

The promoter of interest was fused to the promoter-less lac $Z$ on the reporter plasmids pKAGb-2(-), followed by conjugation into A. hydrophila mutant and wild type strains as described elsewhere (Tabei et al., 2009). Activity of the promoters were measured as a function of $\beta$-galactosidase activity. A. hydrophila cultures were grown in triplicate to an OD600 of $0.5-0.8$ and were then chilled on ice for $15 \mathrm{~min}$. Duplicate assays were performed at $30^{\circ} \mathrm{C}$ on $200 \mu \mathrm{l}$ of cells for each culture in a total volume of $1 \mathrm{ml}$ following permeabilization of the cells with chloroform sodium-dodecyl sulfate (Miller, 1972). Values are presented in Miller units (MU).

\section{Protein Over-Expression and Purification}

The E. coli protein expression strain (BL21Star or NEB ER2523 Express) containing the plasmid of interest (pET28a or pMALc $5 \mathrm{x}$ constructs respectively) was incubated overnight in $10 \mathrm{ml} \mathrm{LB}$ broth with appropriate antibiotics and 1\% (w/v) filter sterilized glucose. On the next day, $1 \mathrm{ml}$ of the culture was transferred into $1 \mathrm{~L}$ of $\mathrm{LB}$ broth with appropriate antibiotics and $1 \%$ $(\mathrm{w} / \mathrm{v})$ filter-sterilized glucose. It was then incubated at $37^{\circ} \mathrm{C}$ 


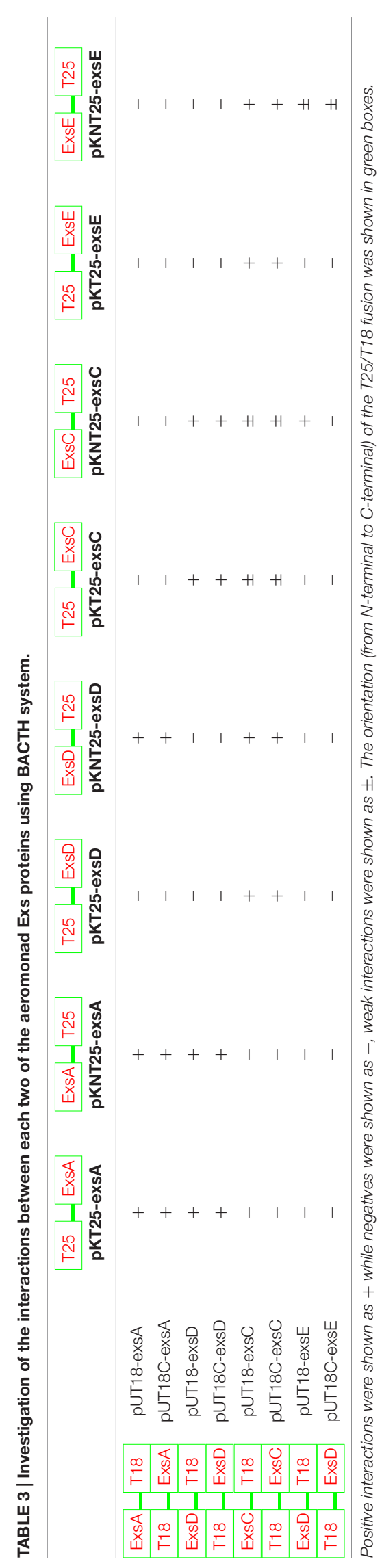

for approximately $2 \mathrm{~h}$ until the $\mathrm{OD}_{600}$ was around 0.2 . At this point, $0.3 \mathrm{mM}-1 \mathrm{mM}$ IPTG was added to induce the protein expression. After $2-3 \mathrm{~h}$ incubation, the cells were harvested by centrifugation at $8,000 \times g$ for $15 \mathrm{~min}$ at $4^{\circ} \mathrm{C}$. The pellet was resuspended in Binding Buffer $[10 \mathrm{mM}$ Imidazole, $500 \mathrm{mM}$ $\mathrm{NaCl}, 5 \%(\mathrm{v} / \mathrm{v})$ Glycerol and $20 \mathrm{mM}$ Phosphate Buffer] for Histidine-tagged proteins or in Column Buffer $(20 \mathrm{mM}$ Tris- $\mathrm{HCl}$ pH7.4, $200 \mathrm{mM} \mathrm{NaCl}, 1 \mathrm{mM}$ EDTA, $1 \mathrm{mM}$ sodium azide and $10 \mathrm{mM} \beta$-mercaptoethanol) for MBP-tagged proteins and frozen overnight at $-20^{\circ} \mathrm{C}$. The pellet was then sonicated using Jencons Vibracell at $20 \mathrm{kHz}$ (20\% amplitude) for $8 \mathrm{x} 20 \mathrm{~s}$ with $1 \mathrm{~min}$ intervals on ice. The sonicated sample was then centrifuged at $30,000 \times g$ for $30 \mathrm{~min}$ at $4^{\circ} \mathrm{C}$ to separate soluble and insoluble proteins. After sonication, the supernatant that contained soluble proteins was transferred into a fresh universal tube while the pellet was stored at $-20^{\circ} \mathrm{C}$. Histidine-tagged proteins (pET28a constructs) were purified using $1 \mathrm{ml}$ HisTrap HP Column (GE Healthcare) as recommended by the supplier. While MBPtagged proteins (pMAL-c5x constructs) were purified using $1 \mathrm{ml}$ MBPTrap HP columns (GE Healthcare) according to the pMAL Protein Fusion and Purification System manual (New England BioLabs). Purification to approximately $95 \%$ was assessed by SDS-PAGE.

\section{Western Blot and Far-Western Blot}

Proteins were separated by SDS-PAGE and transferred onto a nitrocellulose membrane $(0.45 \mu \mathrm{m}$ pore size). The membrane was then blocked in $20 \mathrm{ml}$ of PBS $+5 \%(\mathrm{w} / \mathrm{v})$ dry skimmed milk powder for $1 \mathrm{~h}$. After blocking, the membrane was washed $10 \mathrm{~min}$ in $20 \mathrm{ml}$ PBS Buffer with $0.1 \%(\mathrm{v} / \mathrm{v})$ Tween20 (PBST). Then $10 \mu \mathrm{l}$ (1:2000) of primary antibody (anti-penta-His [Qiagen] for His-tagged proteins and anti-MBP [New England BioLabs] for MBP-tagged proteins) was added to $20 \mathrm{ml}$ of PBST $+5 \%$ milk, in which the membrane was soaked for $1 \mathrm{~h}$. After primary antibody binding, the membrane was washed three times $10 \mathrm{~min}$ in $20 \mathrm{ml}$ PBST Buffer. Then the membrane was probed with $20 \mathrm{ml} \mathrm{PBST}+5 \%$ milk with $5 \mu \mathrm{l} \mathrm{HRP-conjugated} \mathrm{secondary}$ antibody (1:4000) for $1 \mathrm{~h}$. After secondary antibody binding, the membrane was washed three times $10 \mathrm{~min}$ in $20 \mathrm{ml}$ PBST Buffer again and was developed by Pierce ECL Western Blotting and Chemidoc XRS+ System (Bio-Rad).

Far-Western blotting was based on western blotting and used to investigate protein-protein interactions. In Far-Western Blots, the target proteins were probed with a putative interacting protein, which had a different tag to the target protein. The interactions between two proteins could then be detected by Western Blot using antibodies against the tag on the putative interacting protein. After the transfer of proteins and blocking, the nitrocellulose membrane was probed with a non-antibody protein with a different fusion tag to the loaded proteins. The membrane was probed for $1 \mathrm{~h}$ followed by three times $10 \mathrm{~min}$ wash with PBST Buffer. Then the membrane was then incubated with the primary antibody against the tag on the probing protein for $1 \mathrm{~h}$ followed by three times 10 min wash with PBST Buffer. This was followed by a conjugated secondary antibody against the primary antibody and following washing the membrane was developed as above. 


\section{Bacterial Adenylate Cyclase Two-Hybrid (BACTH) Assay}

The BACTH Assay was carried out using the Euromedex BACTH System Kit. The target genes were first cloned into the BACTH plasmids (pKT25, pKNT25, pUT18 and pUT18C) in frame with either T25 or T18 fragments of the cyaA gene at the $5^{\prime}$ or $3^{\prime}$ end of the gene of interest (Table 2), to allow co-expression of fusion proteins. Then one of the T25 derived plasmid constructs was cotransformed with a T18 derived plasmid construct into E. coli BTH101 reporter cells and incubated on MacConkey/maltose agar $\left[4 \%(\mathrm{w} / \mathrm{v})\right.$ MacConkey agar base (Difco $\left.{ }^{\mathrm{TM}}\right), 1 \%(\mathrm{w} / \mathrm{v})$ maltose, $0.5 \mathrm{mM}$ IPTG and appropriate antibiotics) for 2 nights at $30^{\circ} \mathrm{C}$.

When the two proteins of interest interacted with each other, heterodimerization of the fusion proteins allowed the complementation of the T25 and T18 fragments to form a catalytic domain of adenylate cyclase (CyaA), thus cAMP was synthesized. Then the mal operon was activated by cAMP/CAP complex. Therefore the maltose metabolism pathway was switched on in the E. coli BTH101 reporter strain, which allowed the fermentation of maltose and the production of acid that turned the $\mathrm{pH}$ indicator in MacConkey agar pink. Therefore positive interaction colonies were pink/red in color whereas negative colonies were white.

\section{RESULTS}

\section{Mutation of ExsD Affects Swarming But Not Swimming Motility in A. hydrophila}

In order to isolate mutants involved in swarming motility that were encoded outside the lateral flagella gene cluster, random transposon mutagenesis was performed. Over 2000 mutants generated by this method were qualitatively screened for reduced or increased swarming on swarm plates. Several mutant strains consistently exhibited reduced swarming when compared to the wild type, however, their swimming ability in motility agar remained the same as the wild type. In two of the mutants (A5 and A25) the transposon had inserted into $3^{\prime}$ end of the exsD gene of the aeromonad type III secretion system (T3SS). As the aeromonad T3SS is very similar to that found in $P$. aeruginosa (Vilches et al., 2004) the aeromonad exsD gene encodes a protein that is the anti-activator of ExsA the major transcriptional regulator of the T3SS.

\section{Exs Proteins Modulate T3SS Promoter Activity}

The regulator ExsA usually works as an activator of T3SS although there is some evidence that it can act as a repressor of other systems such flagella. In order to test whether the mutations in the exs $D$ gene are allowing ExsA to act directly or indirectly as a repressor we created individual mutations in the putative regulator and anti-activator genes of the aeromonad T3SS system, namely exs $A, \operatorname{exs} D$, exs $C$, and exsE.

Firstly to investigate the role of the genes in the aeromonad T3SS, the promoter regions of the T3SS operons (Vilches et al., 2004, 2009) were isolated and fused to the promoter-less lacZ gene in the transcriptional reporter plasmid pKAGb-2(-). The promoter activities of a selection of the promoter constructs were then investigated in the A. hydrophila wild type and $\operatorname{exs} A$, exs $C$, exs $D$ and exsE mutant strains. The individual reporter plasmids were introduced into the A. hydrophila strains and the promoter activities measured. The activity of promoter $\mathrm{PascN}$ in the $A$. hydrophila wild type was $808 \mathrm{MU}$, this was reduced in the exs A mutant, in which the promoter activity was down to $15 \mathrm{MU}$ (Figure 1A). In the exs $D$ mutant, the PascN promoter activity

\section{A}

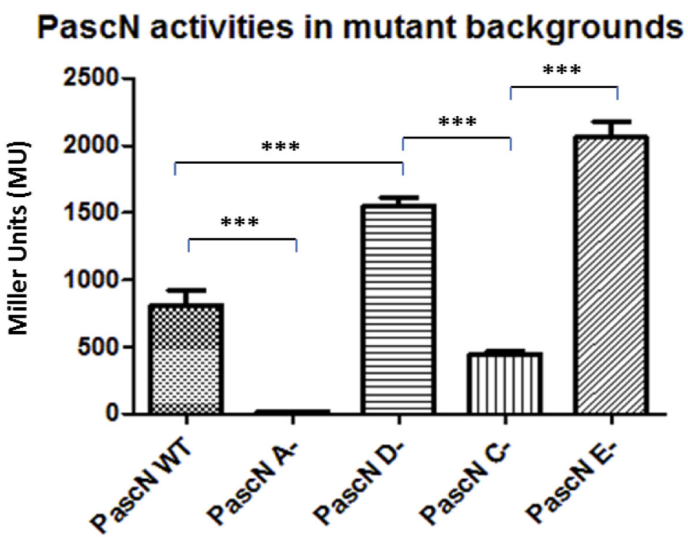

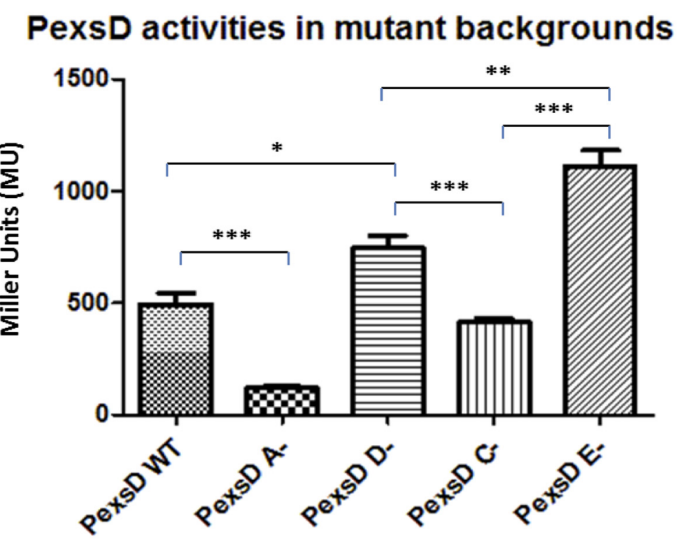

FIGURE 1 | $\beta$-galactosidase activities of promoters PascN (A) and Pexs $D(B)$ in $\boldsymbol{A}$. hydrophila AH3R wild type and mutant backgrounds. The promoter activities were measured when each strain was grown in LB broth to log phase at $30^{\circ} \mathrm{C}$ with shaking at $200 \mathrm{rpm}$. The promoter activities of $P$ asc $N$ and $P$ exs $D$ were significantly decreased in the exsA (A-) mutant and exsC (C-) mutant when compared to the promoter activities in exs $D$ (D-) mutant and exsE (E-) mutant ( $p<0.001)$ Also, the promoter activities in the wild type strain were significantly lower than both the exs $D$ mutant and the exsE mutant strains ( $p<0.001)$. Assays were carried out in triplicate and the values are the means. The error bars showed Standard Error of the Mean (SEM). The graph was created using GraphPad Prism 5. The significance was determined using Student's $t$-test $\left({ }^{*} p<0.05,{ }^{* *} p<0.01,{ }^{* * *} p<0.001\right)$. 
increased to $1551 \mathrm{MU}$, which was an increase from the activities in both wild type and exs A mutant strains $(p<0.001)$. The PascN promoter activity was repressed, but not completely shut down, to $444 \mathrm{MU}$ in the $A$. hydrophila exs $C$ mutant (Figure 1A). While in the A. hydrophila exsE mutant strain, the promoter activity was further increased to $2059 \mathrm{MU}$, which was a significant increase compared to the activities in the A. hydrophila exs $A$ and exs $C$ mutants $(p<0.001)$.

The promoter PexsD showed a similar trend of activities as for the PascN promoter. In the A. hydrophila wild type strain PexsD activity was $494 \mathrm{MU}$ and it was significantly reduced to $121 \mathrm{MU}$ when exsA gene was knocked out $(p<0.001)$ (Figure 1B). In the A. hydrophila exsD mutant, the promoter activity of PexsD increased to $744 \mathrm{MU}$, which was higher than the promoter activity measured in the wild type strain $(p<0.05)$. While in the exs $C$ mutant the promoter activity was reduced to $413 \mathrm{MU}$, which was close to the wild type value but significantly lower than in the exsD mutant strain $(p<0.001)$. When exsE was absent, the promoter activity of Pexs $D$ reached $1109 \mathrm{MU}$, which was a significant increase compared to the activities in all other backgrounds $(p<0.01)$ (Figure 1B). The patterns of the promoter activities in the different mutant backgrounds suggest a modulating regulatory cascade similar to that of $P$. aeruginosa (Diaz et al., 2011).

The promoter Pexs A, which was responsible for the expression of the putative T3SS master regulator ExsA in A. hydrophila, the promoter activity was much higher than the other promoters of the T3SS. In the wild type the PexsA promoter activity was 10,066 MU. Unlike the other promoters of the T3SS, all of which were repressed when exs $A$ was knocked out, the promoter activity of Pexs $A$ was not reduced but demonstrated a significant increase in activity in the exs $A$ mutant to $15,546 \mathrm{MU}(p<0.001)$ (Figure 2). More surprisingly, the promoter activity of PexsA decreased to 5,030 MU in the exsD mutant background, which was significantly lower than the promoter activities of both the wild type and the exs $A$ mutant strains $(p<0.001)$. In the exs $C$ mutant, the promoter activity of Pexs $A$ increased to $12,826 \mathrm{MU}$, which was significantly higher than both the activities in the wild type $(p<0.05)$ and in the exsD mutant strains $(p<0.001)$, but not significantly different from the promoter activity in the exs $A$ mutant. The promoter activity of Pexs $A$ in the exsE mutant was 7,860 MU, which was significantly lower than both the activities in the exs $A$ mutant $(p<0.001)$ and in the exsD mutant strains $(p<0.01)$ (Figure 2).

\section{ExsA Negatively Regulates Its Own Promoter}

Due to the unexpected results of the PexsA promoter activities in the different $A$. hydrophila backgrounds, another approach was required to investigate the regulation of the T3SS. Thereby, E. coli was utilized as a heterologous host to re-constitute T3SS regulation by co-transforming two plasmids into this cell background. The reporter plasmid pKAGb-2(-) containing the aeromonad T3SS promoters fused to $l a c Z$, and the gene encoding ExsA the putative master regulator of the A. hydrophila T3SS, was cloned into the broad host range pBBR1MCS-5 plasmid.

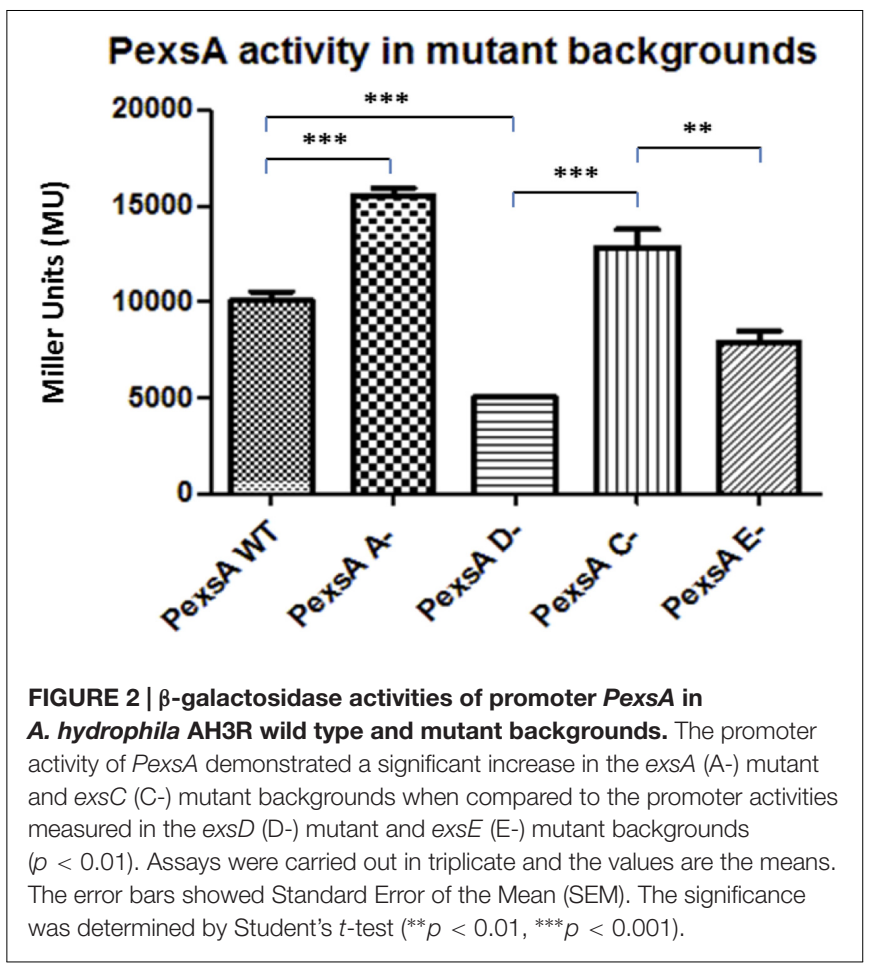

Both plasmids were co-transformed into E. coli and assayed for $\beta$-galactosidase activity.

Each of the T3SS promoter activities was measured in the presence or absence of exsA in E. coli cells by co-transforming the reporter plasmid with pBBR5exsA or empty pBBR1MCS-5 respectively (Figure 3). All of the T3SS promoters, except for promoter PexsA, had very low activity in E. coli, whether or not exs $A$ was present in-trans. When the promoter activity of Pexs $A$ was measured with empty pBBR1MCS-5, the $\beta$-galactosidase activity was approximately 2,806 MU, but when measured with pBBR5exsA construct, the promoter activity of PexsA had decreased to approximately 1,962 MU ( $p<0.001)$ (Figure 3). This agreed with our findings of Pexs $A$ promoter activities in A. hydrophila that the master regulator ExsA negatively regulates its own promoter.

\section{Demonstration of ExsA-ExsD, ExsD-ExsC, and ExsC-ExsE Protein-Protein Interactions}

Extrapolation from other related bacterial T3SS indicate the master regulator of the T3SS in the A. hydrophila is ExsA (Diaz et al., 2011). This protein is able to self-interact for cooperative activation of the T3SS promoters as well as interacting with the anti-activator ExsD, while not interacting with ExsC or ExsE (Diaz et al., 2011). It is not known if a regulatory cascade similar to that of $P$. aeruginosa occurs in A. hydrophila. Therefore a bacterial two-hybrid approach was utilized to investigate the potential protein-protein interactions between the aeromonad Exs proteins. Each exs gene was inserted into all four bacterial two-hybrid plasmid vectors. All combinations are shown in 


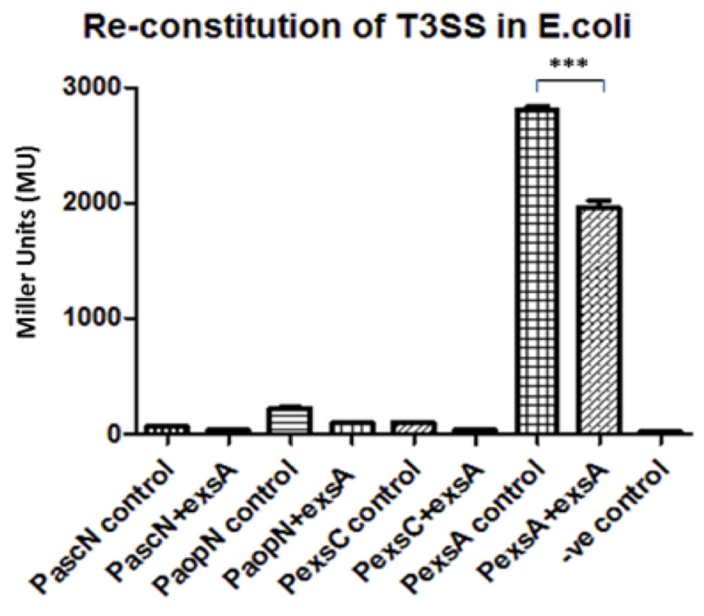

FIGURE 3 | Re-constitution of T3SS regulation in E. coli $\mathrm{DH} 5 \alpha$. The promoters $(P)$ of the aeromonad T3SS were cloned into reporter plasmid pKAGb-2(-) and fused upstream of a promoter-less lacZ gene. The A. hydrohphila AH3R exsA gene was cloned into pBBR1MCS-5 plasmid and co-transformed into $E$. coli DH5 $\alpha$ with pKAGb-2(-) reporter plasmid constructs. $\beta$-galactosidase assays were carried out to measure the promoter activity in the presence or absence of exSA in-trans. Each T3SS promoter activity was measured in the presence of exsA (+exsA) or absence of exs $A$ (control). Negative control (-ve) was measured using promoter-less pKAGb-2(-) plasmid. All of the A. hydrophila T3SS promoters had very low activities in the $E$. coli re-constitution system except for the promoter PexsA. The promoter activity of Pexs $A$ was significantly higher in the absence of exs $A$ rather than in the presence of exsA in-trans $(p<0.001)$. Assays were carried out in triplicate and the values are the means. The error bars showed Standard Error of the Mean (SEM). The significance was determined using Student's $t$-test $(* * * p<0.001)$.

Table 3. These plasmid combinations were used to co-transform the reporter strain E. coli BTH101. ExsA-ExsA interactions were demonstrated with co-transformation of pKT25-exsA with either pUT18-exsA or pUT18C-exsA, and with pKNT25-exsA with pUT18-exsA or pUT18C-exsA (Figure 4A; Table 3). Each of the exsA-fused BACTH plasmid constructs showed positive interactions when co-transformed with each of the exsD-fused BACTH plasmid constructs (Figure 4B). However, ExsA showed no interaction with ExsC or ExsE (Figures 4C,D; Table 3).

ExsD which had been shown to interact with ExsA, showed no evidence of ExsD-ExsD self-interaction. However, ExsD was shown to interact with ExsC in every combination, while no evidence of ExsD-ExsE interaction was found (Table 3).

ExsC did not interact with ExsA in any combination. Strong ExsC-ExsD interactions were shown in every case, while weak interactions were observed for ExsC-ExsC self-interaction due to weaker colouration than seen with ExsC-ExsD or ExsCExsE. Very strong ExsC-ExsE interaction was observed in every combination of exsC-fused BACTH plasmid constructs with exsE-fused BACTH plasmids (Table 3).

ExsE was shown to interact with ExsC only, the exsE-fused BACTH plasmids showed no positive interaction with either ExsA or ExsD. As demonstrated in the ExsC interactions, ExsE-ExsC interactions were very strong in every case. A weak ExsE-ExsE self-interaction was observed (Table 3).
To confirm the interaction results from the bacterial twohybrid assay, the Exs proteins were further investigated by Far western blot. In order to facilitate this each protein was expressed and purified as both His-tagged and MBP-fused versions.

The purified four His-tagged Exs proteins were loaded on an SDS-PAGE gel (Figure 5A) and blotted together with an MBP-fused ExsC as a positive control. When probed with the MBP5 kit control protein (without any Exs fusion) only the positive control MBP-ExsC fusion protein (and degradation products) was detected, demonstrating that the His-tagged Exs proteins were unable to interact with the MBP5 negative control (Figure 5B).

Only the ${ }_{H i s}$ ExsD protein was detected in the Far-Western Blot when probed with MBP-ExsA (Figure 5C) indicating the interaction between ExsA and ExsD. No interaction with ExsC or ExsE was seen. Furthermore, no self-interaction with ExsA was observed (Figure 5C), contrary to the findings of the BACTH assay.

When MBP-ExsD was used to probe the His-tagged Exs proteins, no bands were observed except for the positive control (data not shown). Therefore, the Far-Western Blot was carried out in reverse, using His ExsD to probe the MBP-fused Exs proteins. Only the MBP-ExsC protein was detected (and the positive control), showing the interaction between ExsD and ExsC (Figure 5D).

When MBP-ExsC was used as a probe, both the ${ }_{H i s}$ ExsD and His ExsE proteins were detected (Figure 5E). There were two bands of different sizes detected for ${ }_{H i s}$ ExsD, one was the size of the ${ }_{H i s} \operatorname{ExsD}(\sim 33 \mathrm{kDa})$ while the other one was possibly the size of dimerized ${ }_{\text {His }}$ ExsD ( $\left.66 \mathrm{kDa}\right)$. This demonstrates ExsC-ExsD interaction and ExsC-ExsE interaction. No interaction was seen for ExsA or self-interaction with ExsC (Figure 5E).

The ${ }_{H i s}$ ExsC and the ${ }_{H i s}$ ExsE proteins were detected when MBP-ExsE was used as a probe (Figure 5F) demonstrating interactions between ExsE and ExsC as well as the ExsE-ExsE self-interaction.

\section{Mutations in the T3SS Regulatory System Affect Swarming Motility}

As the results presented here suggest the presence of a T3SS regulatory cascade of Exs proteins in A. hydrophila, further investigation of the effect of the exs mutations on swarming motility in A. hydrophila were carried out. Different strains of $A$. hydrophila including the exs $A$ mutant, exs $C$ mutant, exs $D$ mutant, exsE mutant, and a lafK mutant were plated on the swarming agar together with the wild type. The wild type strain was able to swarm on the surface of the $0.6 \%(\mathrm{w} / \mathrm{v})$ semi-solid agar, but when the major regulator of the Aeromonas lateral flagella system was knocked out in the lafK mutant, swarming motility was completely lost. Moreover, the swarming motility was not affected in the exs $A$ mutant or exs $C$ mutant as the mutant strains were able to swarm as much as in wild type strain. However, when the exs $D$ gene or exsE gene were mutated, the swarming motility was reduced.

The phenotypes of the swarming assays were quantified by measuring the swarming diameters of each strain (Figure 6). The 
A pKT25-exsA + pUT18C-exsA
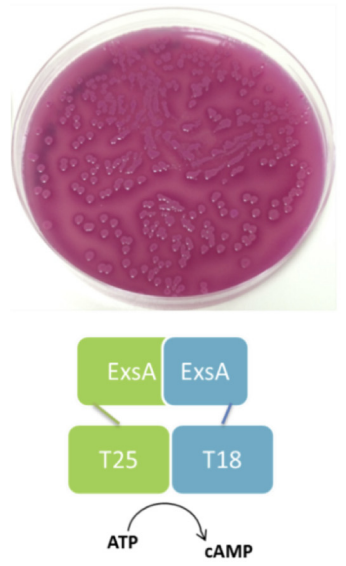

B pKT25-exsA + pUT18C-exsD
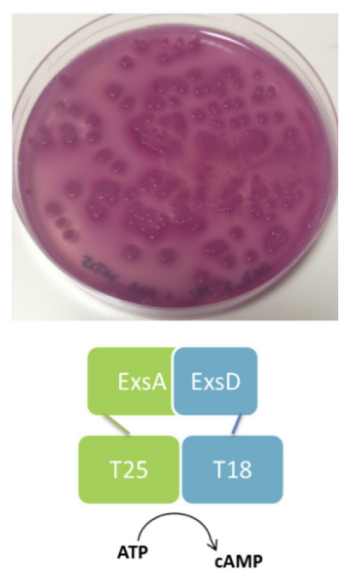

C pUT18-exsA + pKNT25-exsC
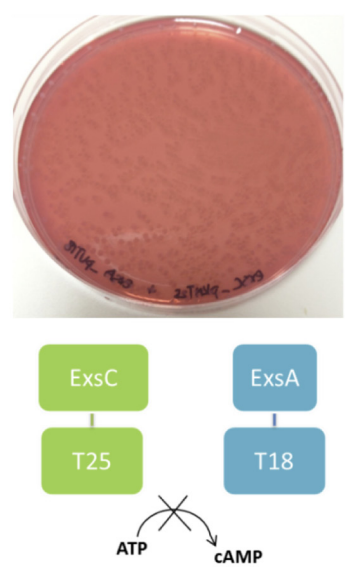

D pUT18C-exsA + pKNT25-exsC
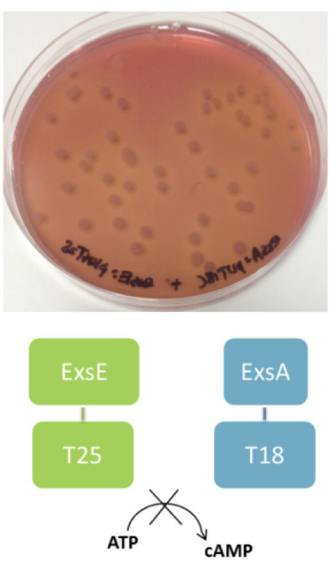

FIGURE 4 | Illustration of BACTH interactions between ExsA and each of the Exs proteins. Plasmid combinations were co-transformed into E. coli BTH101 and grown on MacConkey-maltose agar for 2 days at $30^{\circ} \mathrm{C}$. (A) pKT25-exsA with pUT18C-exsA showing strong ExsA-ExsA self-interaction (B), pKT25-exsA with pUT18C-exsD showing strong ExSA-ExsD interaction; (C), pUT18-exsA with pKNT25-exsC showing no interaction between ExsA and ExSC; (D), pUT18C-exsA with pKNT25-exsE showing no interaction between ExsA and ExsE. The BACTH assay was carried out three times for each combination.

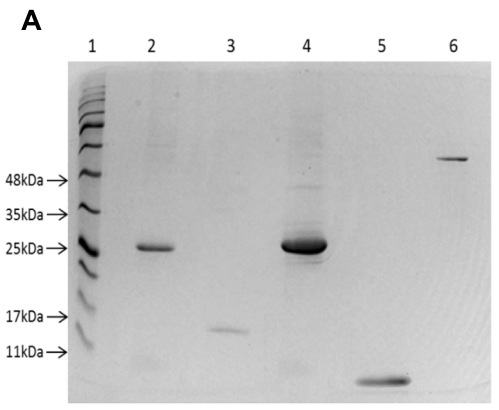

D

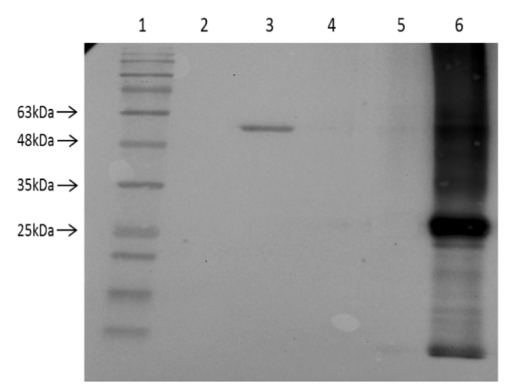

B

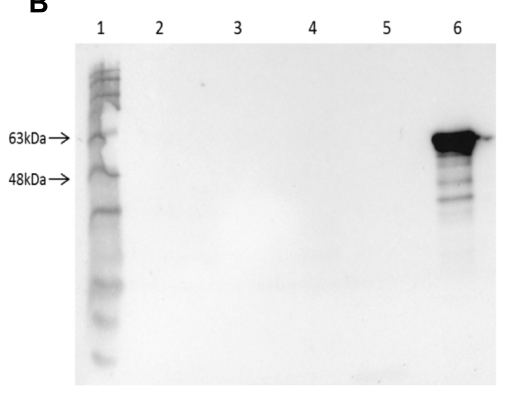

E

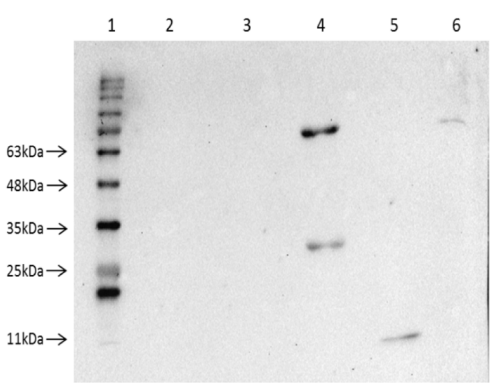

C

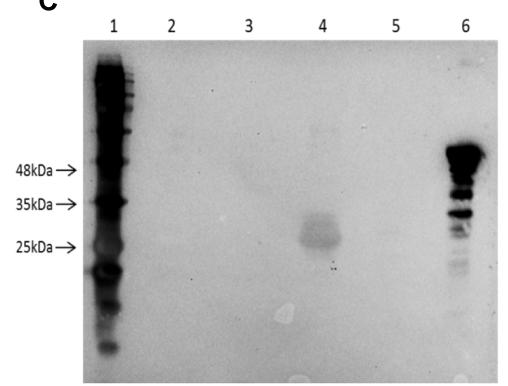

$\mathbf{F}$

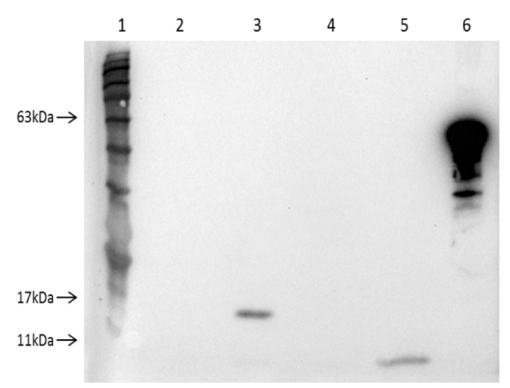

FIGURE 5 | Investigation of the Exs protein interactions using Far-Western Blot. (A) A 12\% SDS-PAGE gel showing the purified Exs proteins and a MBP-fused Exs protein as positive control for Far-Western Blot. Lane 1, BLUeye Prestained Protein Ladder (Geneflow); Lane 2, purified His ExsA protein ( 33 kDa);

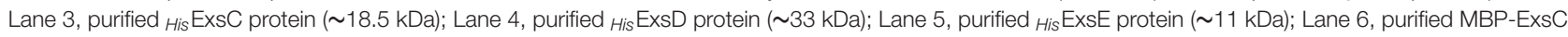
protein $(\sim 61 \mathrm{kDa})$. The proteins on this SDS-PAGE gel were then transferred onto a nitrocellulose membrane for Far-Western Blot. (B) Negative control Far-Western Blot of the control protein MBP5 probing His-tagged Exs proteins. Only MBP-ExSC was observed after probing, suggesting that MBP5 protein without Exs fusions was unable to interact with His-tagged Exs proteins. (C) Far-Western Blot of MBP-ExsA probing His-tagged Exs proteins. The His ExsD protein was detected, suggesting ExsA-ExsD interaction. (D) Far-Western Blot of ${ }_{\text {His }}$ ExsD probing MBP-fused Exs proteins. Only MBP-ExSC ( 61 kDa) was detected when probed with $H_{i i s}$ ExsD protein. (E) Far-Western Blot of MBP-ExsC probing His-tagged Exs proteins. The ${ }_{H i s}$ ExsD $_{\text {and }}$ His ExsE proteins were detected, suggesting ExsC-ExsD and ExsC-ExsE interactions. The upper band showing in lane 4 was possibly the size of a dimerized His ExSD ( 66 kDa). (F) Far-Western Blot of MBP-ExsE probing His-tagged Exs proteins. The ${ }_{H i s}$ ExsC $_{\text {and }}{ }_{H i s}$ ExsE were detected when probed with MBP-ExsE, suggesting ExsE-ExsC interaction and ExsE-ExsE self-interaction. 


\section{Swarming assay of different AH3R strains}

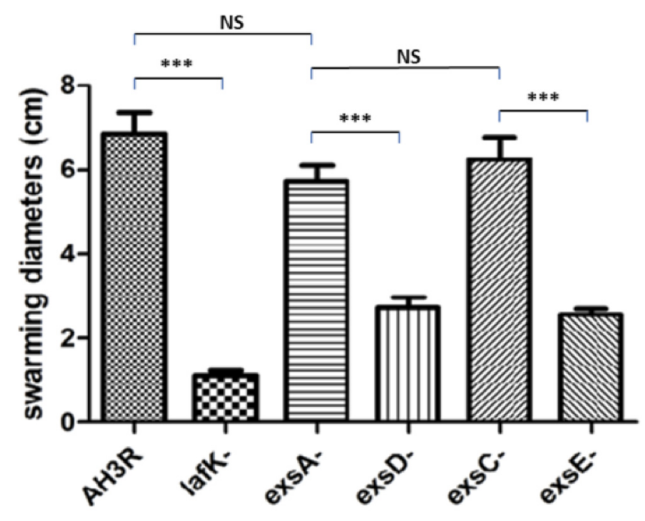

FIGURE 6 | Swarming assays of different mutant strains of A. hydrophila AH3R. Bacteria were incubated on the swarming agar $0.6 \%$ $(\mathrm{W} / \mathrm{V})$ at $30^{\circ} \mathrm{C}$ overnight and the diameter of the swarm measured. The experiment was repeated at least 15 times. Quantification of the swarming assays of $A$. hydrophila AH3R wild type and mutant strains. The swarming diameters were significantly lower in the lafK mutant when compared to the A. hydrophila AH3R wild type $\left({ }^{* * *} p<0.001\right)$, while there was no significant difference of swarming diameters among the wild type strain, the exsA mutant and exs $C$ mutant $(p>0.05)$. However, the swarming diameters of exs $D$ mutant and exsE mutant were significant decreased when compared to the exsA mutant and exsC mutant (*** $p<0.001)$. The error bars showed

Standard Error of the Mean (SEM). The significance was determined using Student's $t$-test.

average swarming diameter of the A. hydrophila wild type strain was $6.9 \mathrm{~cm}$ while it was significantly decreased to $1.1 \mathrm{~cm}$ in the lafK mutant strain $(p<0.001)$. The swarming motility of the exs $A$ and exs $C$ mutant strains was not significantly different from the wild type, with an average swarming diameter of 5.7 and $6.3 \mathrm{~cm}$ respectively $(p>0.05)$. Swarming motility was repressed in exs $D$ and exs $E$ mutant strains when compared to the wild type, and the exs $A$ and exs $C$ mutant strains $(p<0.001)$. The average swarming diameters of exs $D$ mutant and exsE mutant strains were 2.7 and $2.6 \mathrm{~cm}$ respectively, although reduced these were also significantly different from the lafK mutant $(p<0.05)$ (Figure 6).

\section{Exs Regulatory Proteins Modulate Lateral Flagella Promoter Activity}

In order to investigate the effect of the mutations on the aeromonad lateral flagella system, several of the lateral flagella promoter regions (Wilhelms et al., 2013) were fused to lac $Z$ in the promoter probe transcriptional fusion vector pKAGb-2(-) and introduced into A. hydrophila strains. The activities of the lateral flagella promoters were measured in bacteria grown on swarming agar.

Among all putative promoters, PfliM, PflgB and PlafA were chosen, one from each class of the lateral flagella transcriptional hierarchy (Wilhelms et al., 2013). To investigate the potential cross-talk between the lateral flagella system and the T3SS, promoter activities were measured in different mutant backgrounds.
The PfliM promoter activity in the wild type was $247 \mathrm{MU}$ this decreased to $22 \mathrm{MU}$ in the lafK mutant $(p<0.001)$. The promoter activity of PfliM was $215 \mathrm{MU}$ in the exsA mutant but decreased to $88 \mathrm{MU}$ in the exsD mutant strain $(p<0.001)$. Similarly, in the exs $C$ mutant strain, the promoter activity of PfliM was $225 \mathrm{MU}$ and decreased to $143 \mathrm{MU}$ in the exsE mutant strain $(p<0.001)$. There was no significant difference of the promoter activities among the exsA mutant, the exs $C$ mutant and the wild type strains $(p>0.05)$. However, the promoter activity in the exsD mutant was significantly higher than in the lafK mutant while it was significantly lower than in the exsE mutant $(p<0.001)$ (Figure 7A).

The $P f \lg B$ promoter activity was $970 \mathrm{MU}$ in the wild type and decreased to $20 \mathrm{MU}$ in the lafK mutant $(p<0.001)$. The promoter activity was $684 \mathrm{MU}$ in the exs $A$ mutant strain, which was not significantly different with the promoter activities in the wild type and the exsC mutant strain $(p>0.05)$ while it was significantly higher than the promoter activities in the lafK mutant, exsD mutant and exsE mutant strains $(p<0.001)$. The promoter activity of $P f l g B$ was $105 \mathrm{MU}$ in the exsD mutant while it increased to $665 \mathrm{MU}$ in the exsC mutant strain $(p<0.001)$. $P f l g B$ promoter activity was $435 \mathrm{MU}$ in the exsE mutant, which was significantly lower than in the wild type, the exsA mutant and the exs $C$ mutant strains but significantly higher than in the lafK mutant and exsD mutant strains $(p<0.001)$ (Figure 7B).

The pattern of PlafA promoter activity was similar to PfliM and PflgB. In the wild type, PlafA was $976 \mathrm{MU}$ that decreased to $22 \mathrm{MU}$ in the lafK mutant strain (Figure 7C). PlafA promoter activity in the exs $A$ mutant was $771 \mathrm{MU}$, which was significantly higher than in the lafK mutant $(p<0.001)$, the exsD mutant $(p<0.001)$ and the exsE mutant $(p<0.01)$. The promoter activity in the exsD mutant was $240 \mathrm{MU}$, which was approximately fourfold lower than in the wild type strain and threefold lower than in the exs $A$ mutant strain. PlafA activity was $734 \mathrm{MU}$ in the exs $C$ mutant strain, which was not significantly different with the promoter activity in the exs $A$ mutant $(p>0.05)$. Unlike PfliM and $P f l g B$, the promoter activity of PlafA in the exsE mutant strain was $638 \mathrm{MU}$, which was not different with the promoter activity measured in the exs $C$ mutant $(\mathrm{p}>0.05)$ but significantly lower than the exsA mutant $(p<0.01)$. The promoter activity of PlafA in the exsE mutant was also significantly higher than in the exs $D$ mutant $(p<0.001)$ (Figure 7C).

\section{DISCUSSION}

While screening for non-swarming transposon mutants, two were isolated that had a reduction in swarming motility. These were surprisingly mutated in a gene encoding ExsD a protein putatively involved in the regulation of the aeromonad T3SS. This was similar to the results of Yu et al. (2007), who demonstrated that exs $D$ mutants have an effect on aeromonad lateral flagella expression. As little is known about the regulation of the T3SS in Aeromonas, this prompted us to further investigate the regulation of this system and its possible cross-talk with the lateral flagella system. The results of this study suggest that the regulatory cascade of the T3SS which involves the ExsA, 
A

Pflim activities in different mutant backgrounds

NS

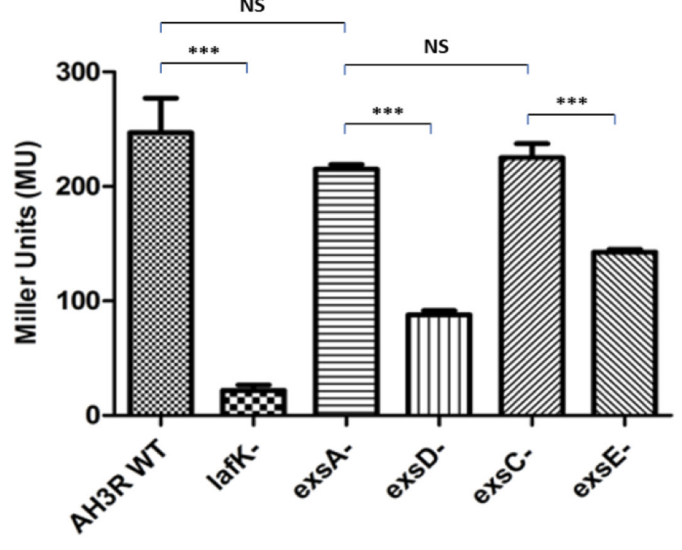

B

PflgB activities in different mutant backgrounds

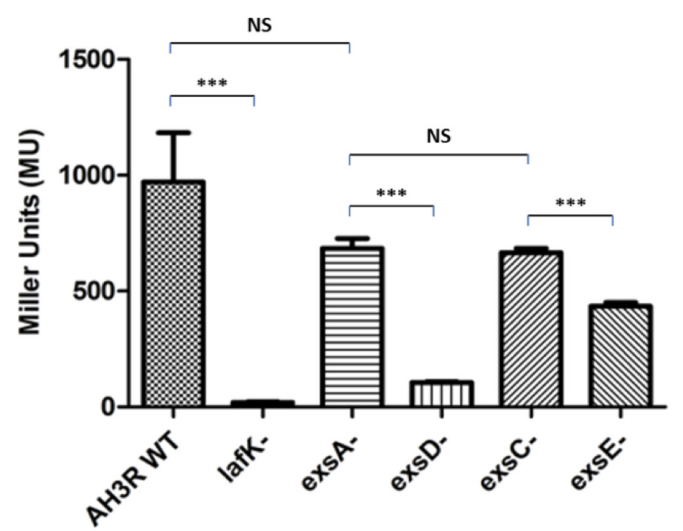

C

PlafA activities in different mutant backgrounds

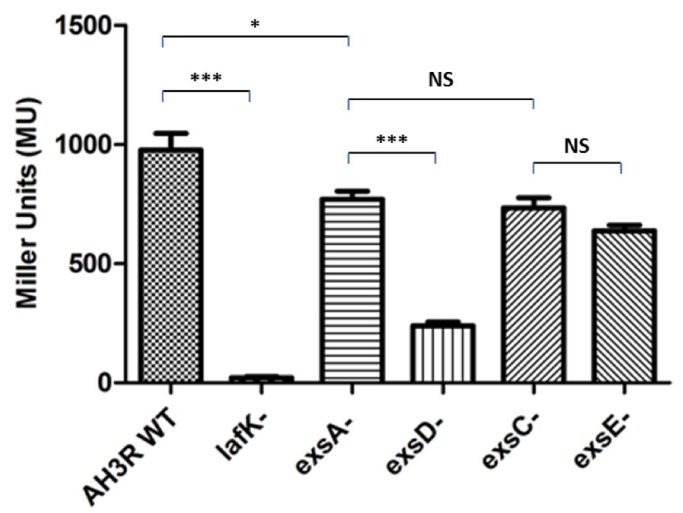

FIGURE $7 \mid \beta$-galactosidase activities of the aeromonad lateral flagella promoters PfliM, PflgB and PlafA in A. hydrophila AH3R wild type and mutant backgrounds. (A) Promoter activities of PfliM were significantly decreased in the lafK mutant, exsD mutant and exsE mutant when compared to the AH3R wild type, exs A mutant and exsC mutant. (B) Promoter activities of PflgB were significantly decreased in the lafk mutant, exs $D$ mutant and exsE mutant when compared to the AH3R wild type, exSA mutant and exsC mutant. (C) Promoter activities of PlafA were significantly decreased in the lafK mutant and exsD mutant when compared to the AH3R wild type, exsA mutant and exSC mutant. While in the exsE mutant, promoter activity of PlafA was significantly lower than in the exsA mutant but at a similar level with the exsC mutant. The promoter activities were measured when each strain was grown on swarming agar at $30^{\circ} \mathrm{C}$ overnight. Assays were carried out in triplicate and the values are the means. The error bars showed Standard Error of the Mean (SEM). The significance was determined using Student's $t$-test $\left(* p<0.05,{ }^{* * *} p<0.001\right)$.

ExsD, ExsC, and ExsE proteins in A. hydrophila is similar to the T3SS regulatory cascade reported in $P$. aeruginosa (Yahr et al., 1995; Diaz et al., 2011). The AraC family transcriptional activator ExsA functions as the master regulator of the T3SS in A. hydrophila, as its mutation reduces expression from all the T3SS promoters with the exception of its own. The anti-activator protein ExsD inhibits the master regulator ExsA through direct protein-protein interactions. The chaperone protein ExsC is able to sequester the anti-activator ExsD from ExsA by direct binding to ExsD. While the effector protein ExsE binds to its cognate chaperone protein ExsC directly thus releasing ExsD to inhibit ExsA. The Exs regulatory cascade is also shown to be involved in the regulation of the lateral flagella system, in which ExsA possibly functions in down-regulating the lateral flagella promoter activities and suppressing the swarming motility.

Before measuring the activities of A. hydrophila T3SS promoters in the mutant backgrounds, the assay conditions were optimized, as there was evidence showing that the expression of the A. hydrophila T3SS was induced significantly with additional $20 \mathrm{mM} \mathrm{MgCl} 2$ and $10 \mathrm{mM}$ EGTA. Vilches et al. (2009) demonstrated that the expression level of two T3SS mediated genes aopN and aexT were up-regulated with additional $\mathrm{MgCl}_{2}$ and EGTA using $g f p$-fusions. However, in this study, the promoter activities of PaopN and PexsA were decreased in these conditions. Moreover, no significant difference 
of promoter activity between the inducing conditions and noninducing conditions was found for promoters $P$ exs $C$ and $P$ exs $D$ (data not shown). Similar results were reported by $\mathrm{Yu}$ et al. (2007).

In this study, as the T3SS promoters were cloned in the multi-copy lacZ-fusion plasmid pKAGb-2(-), this could possibly give rise to a transcriptional factor titration effect. The potential repressors might be 'outnumbered' by the high copy number of the promoter (Guido et al., 2006; Brewster et al., 2014). Although pKAGb-2(-) is not a high copy number vector.

In the Vilches' study, the activity of the PaopN promoter was obtained by measuring the expression level of $g f p$-fusion. The $g f p$ reporter gene was integrated into the A. hydrophila AH3 chromosomal DNA downstream of the PaopN promoter and upstream of the aopN gene. The integration of $g f p$ gene might affect the expression of the downstream operon, in which at least three genes, $a c r R, a c r G$ and $a c r V$ were present all believed to be involved in the low calcium response (Barve and Straley, 1990; Matson and Nilles, 2001; Burr et al., 2003).

Five promoter sequences, PascN, PaopN, PexsC, PexsA and Pexs $D$ were investigated in the T3SS regulon and four of them were shown to have no activity in the absence of ExsA, except for $P \operatorname{exs} A$, which was responsible for the expression of the master regulator ExsA itself. The promoter activity of PexsA was up-regulated in the exsA mutant while in the E. coli reconstitution system the PexsA promoter activity was downregulated with exsA in-trans. This finding indicates that the master regulator ExsA is under control of negative feedback by inhibiting its own promoter. AraC was reported to repress the expression of its own gene araC by binding to its own promoter region (Casadaban, 1976; Hahn and Schleif, 1983). As a member of AraC family proteins, there has been no report of ExsA that it can repress its own transcription so far. However, many members of the AraC repress their own expression, such as XylR, one of the regulators for xylene metabolism in Pseudomonas putida and YbtA, a pesticin receptor regulator in Yersinia pestis (Inouye et al., 1987; Fetherston et al., 1996).

Due to the lack of T3SS in E. coli re-constitution system, of the T3SS promoters, except for PexsA, had low promoter activity, suggesting that these promoters required certain aeromonad or T3SS-specific factors other than ExsA in order for optimal activation. This contributed to the previous suspicion that there might be secondary regulation or regulators other than the master regulator ExsA. The promoter PexsA was still highly active in $E$. coli in the absence of exsA in-trans, thus the promoter was likely to be activated by housekeeping sigma factor $\sigma^{70}$ in E. coli. While in E. coli with induced exsA expression, the promoter activity of PexsA was down-regulated significantly. This confirmed that ExsA negatively regulates its own promoter PexsA. Therefore, PexsA is constantly activated but under a negative feedback control of ExsA. Therefore, it could be deduced that the A. hydrophila maintains a minimal level of T3SS expression in the environment due to the constitutive expression of ExsA. When bacteria encounter host cells, according to our hypothesis, ExsE is secreted and ExsC is free to bind to ExsD releasing ExsA to further activate the T3SS.
Both the BACTH assay and the Far-Western Blot indicate interactions in between ExsA-ExsD, ExsD-ExsC, and ExsCExsE, supporting the hypothesis of the ExsA-ExsD-ExsCExsE regulatory cascade. Moreover, the self-interaction of ExsA protein was observed from BACTH assay while the selfinteraction of the ExsE protein was observed from Far-Western Blot.

In $P$. aeruginosa the C-terminal domain of ExsA has two helix-turn-helix DNA binding motifs but lacks the ability to selfinteract for cooperative binding of DNA (Brutinel et al., 2009). ExsD was shown to inhibit the DNA-binding ability of ExsA by interacting with the N-terminal domain of ExsA, which is involved in the ExsA-ExsA self-interaction (Brutinel et al., 2010). However, in this study, the C-terminal domain of the ExsA protein was shown to interact with both the $\mathrm{N}$-terminal and C-terminal domain versions of itself in the BACTH assay.

When MBP-ExsA was used to probe His-tagged Exs proteins, only His-ExsD was detected although the interaction appeared weak. This correlates with P. aeruginosa that ExsD is found to bind ExsA only as a folding intermediate when these two proteins were synthesized together (Bernhards et al., 2013). Thereby, strong interactions between ExsD and ExsA were observed in vivo using BACTH assay when they were co-expressed whereas weak interactions were shown in vitro using Far-Western Blot.

The self-association of ExsD and ExsC reported in $P$. aeruginosa was not observed in this study (Diaz et al., 2011). As it has been reported in P. aeruginosa, ExsC dimerises to form a 2:2 heterotetramer with ExsD while it binds to ExsE at a 2:1 ratio (Zheng et al., 2007; Bernhards et al., 2013). This might be due to the presence of $\mathrm{T} 25 / \mathrm{T} 18, \mathrm{His}_{6}$ or MBP onto the $\mathrm{N}$-terminal regions of these proteins.

The interactions of ExsC with each of the Exs proteins shown in the BACTH assay revealed a potential hierarchy of ExsC interactions. The potential strongest interaction was observed in ExsC-ExsE, which was a typical T3SS chaperoneeffector interaction described in $P$. aeruginosa (Dasgupta et al., 2004; Urbanowski et al., 2005; Vogelaar et al., 2010). Although in this study the no binding kinetics were carried out, the ExsC-ExsD interaction was thought to be weaker than the ExsC-ExsE interaction but stronger than the ExsC-ExsC selfinteraction from the observation of the BACTH assay. This finding corresponds to the Isothermal Titration Calorimetry studies with binding affinities for ExsC-ExsD (18 nM) and ExsCExsE (1 nM) in P. aeruginosa (Zheng et al., 2007). This suggests that the chaperone protein ExsC prefers to bind to the effector protein ExsE rather than the anti-activator ExsD. However, when the concentration of ExsE decreases, the abundant ExsC proteins bind to ExsD and antagonize the inhibition of ExsD on ExsA.

The swarming assay of the exs mutants has shown reduced swarming motility in exsD mutant and exsE mutant strains similar to the lafK mutant strain. It suggests that the T3SS master regulator ExsA functions directly or indirectly in repressing the expression of the lateral flagella system in A. hydrophila AH3. It correlates with the findings in Vibrio parahaemolyticus, which possess lateral flagella system as well as two T3SSs. The expression level of the lateral flagella gene $f l g B_{L}$ was significantly repressed when over-expressing ExsA and the swarming motility 


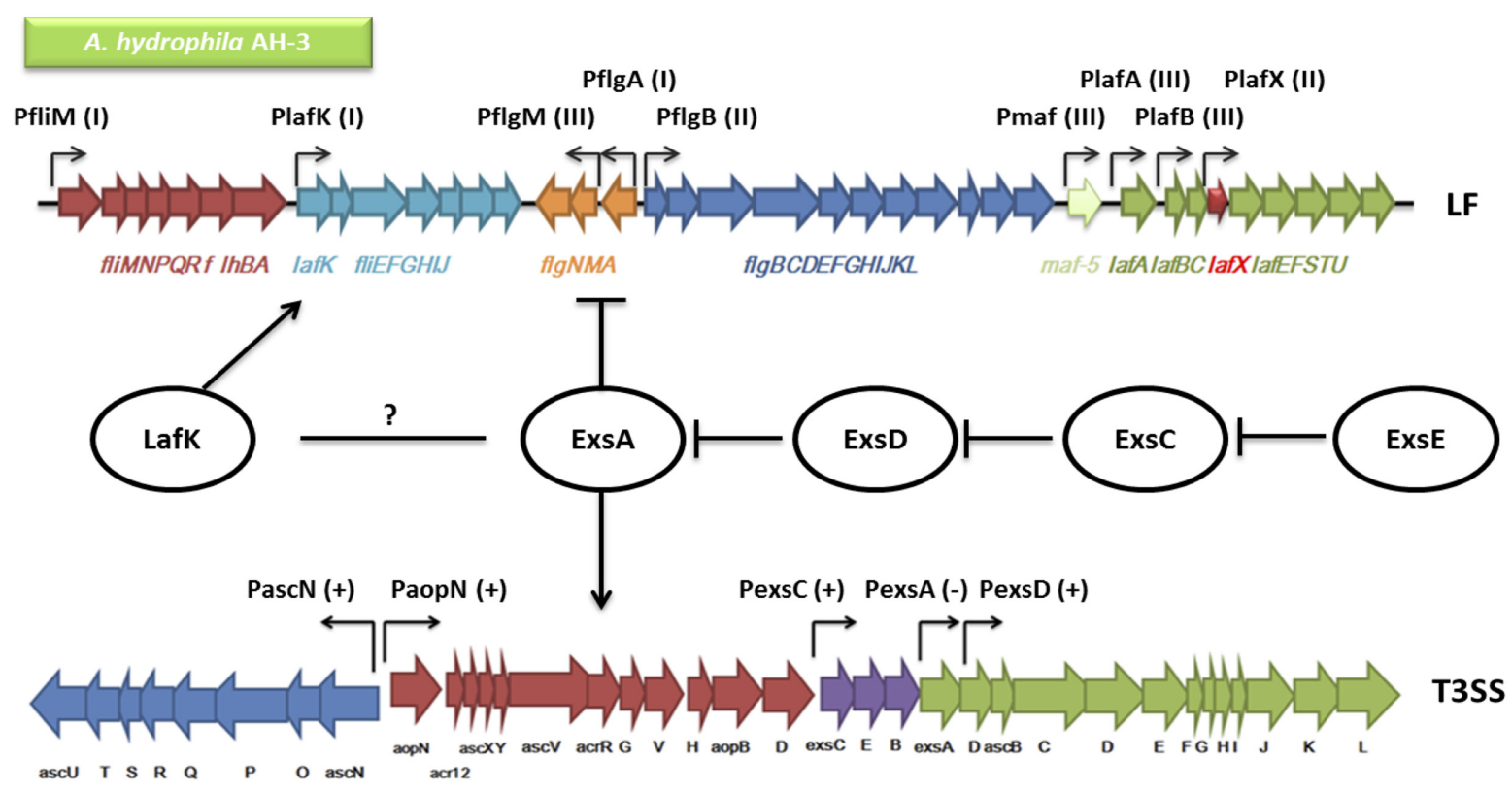

FIGURE 8 | An overview of the genetic regulation between the T3SS and the lateral flagella (LF) system in A. hydrophila AH-3. The regulators including ExSA, ExSD, ExSC, ExsE, and LafK are shown in circles. Each two of the Exs protein are connected by blunt arrows showing the inhibitory interactions in between. The promoters in both the T3SS and the LF system are shown in bent arrows. The promoters in the T3SS are either (+) up-regulated by ExsA or (-) down-regulated by ExsA (PaopN and PexsC data not shown). The LF promoters are categorized into (I) Class I, (II) Class II, or (III) Class III, in which only Class II and Class III promoters are up-regulated by the LF major regulator LafK. The T3SS master regulator ExSA has been shown to down-regulate the expression of the LF system but whether there is interaction between ExSA and LafK is unknown (Genetic alignments of the T3SS and LF were adapted from Vilches et al., 2004 and Canals et al., 2006).

of $V$. parahaemolyticus was inhibited by exs $A$ induction in vivo (Gode-Potratz et al., 2010).

In $V$. parahaemolyticus and A. hydrophila the lateral flagella system is hierarchically controlled at three levels (Canals et al., 2006; Wilhelms et al., 2013).

One lateral flagella promoter was chosen from Class I (PfliM), Class II (PflgB) and Class III (PlafA) and the promoter activities were measured in the wild type, lafK and exs mutant strains in order to investigate the potential cross-talk between the lateral flagella system and the T3SS. The patterns of the promoter activities (PfliM, PflgB and PlafA) measured in the exs mutant backgrounds were similar to the patterns of the swarming diameters of different mutant strains. It indicates that the lateral flagella promoter activities are significantly repressed in the absence of ExsD and ExsE, while not affected in the absence of ExsA and ExsC, supporting the hypothesis that the T3SS master regulator ExsA functions as a repressor of the lateral flagella system. When the anti-activator ExsD is absent, the abundant ExsA proteins suppress the expression of the lateral flagella system. Similarly, when ExsE is absent, the chaperone protein ExsC binds to the anti-activator ExsD thus freeing ExsA to repress the lateral flagella system. There was evidence of crosstalk between the T3SS and the flagella system in $P$. aeruginosa, $Y$. enterocolitica and A. hydrophila AH-1 (Bleves et al., 2002; Soscia et al., 2007; Yu et al., 2007; Vilches et al., 2009). Similar negative cross-talk between the T3SS and the flagella system was determined later in P. aeruginosa as well (Soscia et al., 2007).
In this study the regulatory components of the T3SS were demonstrated to be involved in the regulation of the lateral flagella system in A. hydrophila. Whether the lateral flagella system affects the T3SS in A. hydrophila is still unknown, although there is evidence that the lateral flagella regulator LafK is required for T3SS1 expression in V. parahaemolyticus (GodePotratz et al., 2010).

The overall results correlate with the findings in $V$. parahaemolyticus, which possesses the lateral flagella system and two T3SSs (T3SS1 and T3SS2). It was reported by GodePrtratz et al. (2010) that the expression level of the lateral flagella gene $f l g B L$ was significantly repressed when over-expressing ExsA, and the swarming motility of $V$. parahaemolyticus was inhibited by exsA induction in vivo.

However, it is still unclear how ExsA represses the expression of the lateral flagella system, since the lateral flagella promoter activities were decreased in the absence of ExsD despite the promoter class. The Class I promoter PfliM, the Class II promoter $P f l g B$ and the Class III promoter PlafA were all affected by the absence of ExsD and ExsE. The fact that the Class I promoter is affected suggests that the ExsA protein represses the expression of the lateral flagella system prior to the lateral flagella major regulator LafK.

A schematic overview of a model of the genetic regulation between the T3SS and the lateral flagella system is shown in Figure 8. The T3SS master regulator ExsA activates the transcription of the T3SS by inducing the T3SS promoter 
activities, except that ExsA negative regulates its own promoter PexsA. The master regulator ExsA is controlled by a cascade of proteins including ExsD, ExsC, and ExsE. The anti-activator ExsD inhibits ExsA by direct binding. ExsC inhibits ExsD and ExsE inhibits ExsC via direct protein-protein interactions as well. ExsA was also shown to negatively regulate the lateral flagella system since the absence of ExsD or ExsE represses the swarming ability and the activities of lateral flagellar promoters.

\section{AUTHOR CONTRIBUTIONS}

All authors have made substantial, direct and intellectual contribution to the work and approved it for publication. Y-HZ performed the experiments and helped write the manuscripts. JS

\section{REFERENCES}

Abby, S. S., and Rocha, E. P. C. (2012). The non-flagellar type III secretion system evolved from the bacterial flagellum and diversified into host adapted systems. PLoS Genet. 8:e1002983. doi: 10.1371/journal.pgen.1002983

Barve, S. S., and Straley, S. C. (1990). LcrR, a low-Ca2(+)-response locus with dual Ca2(+)-dependent functions in Yersinia pestis. J. Bacteriol. 172, 4661-4671.

Bernhards, R. C., Marsden, A. E., Esher, S. K., Yahr, T. L., and Schubot, F. D. (2013). Self-trimerization of ExsD limits inhibition of the Pseudomonas aeruginosa transcriptional activator ExsA in vitro. FEBS J. 280, 1084-1094. doi: 10.1111/febs.12103

Bleves, S., Marenne, M. N., Detry, G., and Cornelis, G. R. (2002). Up-regulation of the Yersinia enterocolitica yop regulon by deletion of the flagellum master operon flhDC. J. Bacteriol. 184, 3214-3223. doi: 10.1128/JB.184.12.32143223.2002

Brewster, R. C., Weinert, F. M., Garcia, H. G., Song, D., Rydenfelt, M., and Phillips, R. (2014). The transcription factor titration effect dictates level of gene expression. Cell 156, 1312-1323. doi: 10.1016/j.cell.2014.02.022

Brutinel, E. D., Vakulskas, C. A., and Yahr, T. L. (2009). Functional domains of ExsA, the transcriptional activator of the Pseudomonas aeruginosa type III secretion system. J. Bacteriol. 191, 3811-3821. doi: 10.1128/JB.00002-09

Brutinel, E. D., Vakulskas, C. A., and Yahr, T. L. (2010). ExsD inhibits expression of the Pseudomonas aeruginosa type III secretion system by disrupting ExsA self-association and DNA binding activity. J. Bacteriol. 192, 1479-1486. doi: 10.1128/JB.01457-09

Burr, S. E., Stuber, K., and Frey, J. (2003). The ADP-ribosylating toxin, AexT, from Aeromonas salmonicida subsp. salmonicida is translocated via a type III secretion pathway. J. Bacteriol. 185, 6583-6591.

Canals, R., Altarriba, M., Vilches, S., Horsburgh, G., Shaw, J. G., Tomás, J. M., et al. (2006). Analysis of the lateral flagellar gene system of Aeromonas hydrophila AH-3. J. Bacteriol. 188, 852-862. doi: 10.1128/JB.188.3.852-862.2006

Casadaban, M. J. (1976). Regulation of the regulatory gene for the arabinose pathway, arac. J. Mol. Biol. 104, 557-566. doi: 10.1016/0022-2836(76)90120-0

Cipriano, R. C., and Austin, B. (2011). "Furunculosis and other aeromonad diseases," in Fish Diseases and Disorders: Viral, Bacterial and Fungal Infections, 2nd Edn, Vol. 3, P. T. K. Woo and D. W Bruno (Oxon: CABI Publishing), 424-483.

Cornelis, G. R., and Van Gijsegem, F. (2000). Assembly and function of type III secretory systems. Ann. Rev. Microbiol. 54, 735-774. doi: 10.1146/ annurev.micro.54.1.735

Dasgupta, N., Lykken, G. L., Wolfgang, M. C., and Yahr, T. L. (2004). A novel antianti-activator mechanism regulates expression of the Pseudomonas aeruginosa type III secretion system. Mol. Microbiol. 53, 297-308. doi: 10.1111/j.13652958.2004.04128

de Lorenzo, V., Herrero, M., Jakubzik, U., and Timmis, K. N. (1990). MiniTn5 transposon derivatives for insertion mutagenesis, promoter probing, and chromosomal insertion of cloned DNA in gram-negative eubacteria. J. Bacteriol. 172, 6568-6572. directed the research, had intellectual input into the experimental design and helped write the manuscript.

\section{ACKNOWLEDGMENTS}

We thank Tessabelle Sultana, Wan Ling Wong, and Shengtao Rui for their technical assistance.

\section{SUPPLEMENTARY MATERIAL}

The Supplementary Material for this article can be found online at: http://journal.frontiersin.org/article/10.3389/fmicb. 2016.01434

Diaz, M. R., King, J. M., and Yahr, T. L. (2011). Intrinsic and extrinsic regulation of the type III secretion gene expression in Pseudomonas aeruginosa. Front. Microbiol. 2:89. doi: 10.3389/fmicb.2011.00089

Fetherston, J. D., Bearden, S. W., and Perry, R. D. (1996). YbtA, an AraC-type regulator of the Yersinia pestis pesticin/yersiniabactin receptor. Mol. Microbiol. 22, 315-325. doi: 10.1046/j.1365-2958.1996.00118.x

Frank, D. W., and Iglewski, B. H. (1991). Cloning and sequence analysis of a trans-regulatory locus required for exoenzyme S synthesis in Pseudomonas aeruginosa. J. Bacteriol. 173, 6460-6468.

Gavin, R., Rabaan, A. A., Merino, S., Tomas, J. M., Gryllos, I., and Shaw, J. G. (2002). Lateral flagella of Aeromonas species are essential for epithelial cell adherence and biofilm formation. Mol. Microbiol. 43, 383-397. doi: 10.1046/j.1365-2958.2002.02750

Gibson, D. G., Young, L., Chuang, R. Y., Venter, J. C., Hutchison, C. A. III, and Smith, H. O. (2009). Enzymatic assembly of DNA molecules up to several hundred kilobases. Nat. Methods 6, 343-345. doi: 10.1038/nmeth. 1318

Gode-Potratz, C. J., Chodur, D. M., and McCarter, L. L. (2010). Calcium and iron regulate swarming and type III secretion in Vibrio parahaemolyticus. J. Bacteriol. 192, 6025-6038. doi: 10.1128/JB.00654-10

Gryllos, I., Shaw, J. G., Gavín, R., Merino, S., and Tomás, J. M. (2001). Role of flm locus in mesophilic Aeromonas adherence. Infect. Immun. 69, 65-74. doi: 10.1128/IAI.69.1.65-74.2001

Guido, N. J., Wang, X., Adalsteinsson, D., McMillen, D., Hasty, J., Cantor, C. R., et al. (2006). A bottom-up approach to gene regulation. Nature 439, 856-860. doi: $10.1038 /$ nature 04473

Hahn, S., and Schleif, R. (1983). In vivo regulation of the Escherichia coli AraC promoter. J. Bacteriol. 155, 593-600.

Hanahan, D. (1983). Studies on transformation of Escherichia coli with plasmids. J. Mol. Biol. 166, 557-580. doi: 10.1016/S0022-2836(83)80284-8

Harshey, R. M. (2003). Bacterial motility on a surface: many ways to a common goal. Ann. Rev. Microbiol. 57, 249-273. doi: 10.1146/annurev.micro.57.030502. 091014

Herrero, M., de Lorenzo, V., and Timmis, K. N. (1990). Transposon vectors containing non-antibiotic resistance selection markers for cloning and stable chromosomal insertion of foreign genes in gram-negative bacteria. J. Bacteriol. $172,6557-6567$.

Hovey, A. K., and Frank, D. W. (1995). Analyses of the DNA-binding and transcriptional activation properties of ExsA, the transcriptional activator of the Pseudomonas aeruginosa exoenzyme S regulon. J. Bacteriol. 177, 4427-4436.

Inouye, S., Nakazawa, A., and Nakazawa, T. (1987). Expression of the regulatory gene xylS on the TOL plasmid is positively controlled by the xylR gene product. Proc. Natl. Acad. Sci. U.S.A. 84, 5182-5186. doi: 10.1073/pnas.84.15.5182

Janda, J. M., and Abbott, S. L. (2010). The genus Aeromonas: taxonomy, pathogenicity, and infection. Clin. Microbiol. Rev. 23, 35-73. doi: 10.1128/CMR. 00039-09

Kaniga, K., Delor, I., and Cornelis, G. R. (1991). A wide-host-range suicide vector for improving reverse genetics in gram-negative bacteria: inactivation of the 
blaA gene of Yersinia enterocolitica. Gene 109, 137-141. doi: 10.1016/03781119(91)90599-7

Kirov, S. M. (2003). Bacteria that express lateral flagella enable dissection of the multifunctional roles of flagella in pathogenesis. FEMS Microbiol. Lett. 224, 151-159. doi: 10.1016/S0378-1097(03)00445-2

Kirov, S. M., Castrisios, M., and Shaw, J. G. (2004). Aeromonas flagella (polar and lateral) are enterocyte adhesins that contribute to biofilm formation on surfaces. Infect. Immun. 72, 1939-1945. doi: 10.1128/IAI.72.4.1939-1945. 2004

Kirov, S. M., Tassell, B. C., Semmler, A. B., O’Donovan, L. A., Rabaan, A. A., and Shaw, J. G. (2002). Lateral flagella and swarming motility in Aeromonas species. J. Bacteriol. 184, 547-555. doi: 10.1128/JB.184.2.547-555.2002

Kovach, M. E., Phillips, R. W., Elzer, P. H., Roop, R. M., and Peterson, K. M. (1994). pBBR1MCS: a broad-host-range cloning vector. Biotechniques 16, 800-802.

Lowry, R., Balboa, S., Parker, J. L., and Shaw, J. G. (2014). Aeromonas flagella and colonisation mechanisms. Adv. Microb. Physiol. 65, 203-256. doi: 10.1016/bs.ampbs.2014.08.007

Matson, J. S., and Nilles, M. L. (2001). LcrG-LcrV interaction is required for control of Yops secretion in Yersinia pestis. J. Bacteriol. 183, 5082-5091. doi: 10.1128/JB.183.17.5082-5091.2001

McCarthy, D. H., and Rawle, C. T. (1975). The rapid serological diagnosis of fish furunculosis caused by "smooth" and "rough" strains of Aeromonas salmonicida. J. Gen. Microbiol. 86, 185-187. doi: 10.1099/00221287-86-1-185

McCaw, M. L., Lykken, G. L., Singh, P. K., and Yahr, T. L. (2002). ExsD is a negative regulator of the Pseudomonas aeruginosa type III secretion regulon. Mol. Microbiol. 46, 1123-1133. doi: 10.1046/j.1365-2958.2002.03228

Miller, J. H. (1972). Experiments in Molecular Genetics. Cold Spring Harbor, NY: Cold Spring Harbor Laboratory, 466.

Miller, V. L., and Mekalanos, J. J. (1988). A novel suicide vector and its use in construction of insertion mutations: osmoregulation of outer membrane proteins and virulence determinants in Vibrio cholerae requires toxR. J. Bacteriol. 170, 2575-2583.

Parker, J., and Shaw, J. G. (2011). Aeromonas: clinical microbiology and disease. J. Infect. 62, 109-118. doi: 10.1016/j.jinf.2010.12.003

Rietsch, A., Vallet-Gely, I., Dove, S. L., and Mekalanos, J. J. (2005). ExsE, a secreted regulator of type III secretion genes in Pseudomonas aeruginosa. Proc. Natl. Acad. Sci. U.S.A. 102, 8006-8011. doi: 10.1073/pnas.0503005102

Sha, J., Wang, S. F., Suarez, G., Sierra, J. C., Fadl, A. A., Erova, T. E., et al. (2007). Further characterization of a type III secretion system (T3SS) and of a new effector protein from a clinical isolate of Aeromonas hydrophila-part I. Microb. Pathog. 43, 127-146. doi: 10.1016/j.micpath.2007.05.002

Soscia, C., Hachani, A., Bernadac, A., Filloux, A., and Bleves, S. (2007). Cross talk between type III secretion and flagellar assembly systems in Pseudomonas aeruginosa. J. Bacteriol. 189, 3124-3132. doi: 10.1128/JB.01677-06

Tabei, S. M. B., Hitchen, P. G., Day-Williams, M. J., Merino, S., Vart, R., Pang, P.-C., et al. (2009). An Aeromonas caviae genomic island is required for both O-antigen lipopolysaccharide biosynthesis and flagellin glycosylation. J. Bacteriol. 191, 2851-2863. doi: 10.1128/JB.01406-08

Urbanowski, M. L., Lykken, G. L., and Yahr, T. L. (2005). A secreted regulatory protein couples transcription to the secretory activity of the Pseudomonas aeruginosa type III secretion system. Proc. Natl. Acad. Sci. U.S.A. 102, 99309935. doi: 10.1073/pnas. 0504405102

van Vliet, A. H., Wooldridge, K. G., and Ketley, J. M. (1998). Iron-responsive gene regulation in a Campylobacter jejuni fur mutant. J. Bacteriol. 180, 5291-5298.

Vilches, S., Jimenez, N., Tomas, J. M., and Merino, S. (2009). Aeromonas hydrophila AH-3 type III secretion system expression and regulatory network. Appl. Environ. Microbiol. 75, 6382-6392. doi: 10.1128/AEM.00222-09

Vilches, S., Urgell, C., Merino, S., Chacon, M. R., Soler, L., Castro-Escarpulli, G., et al. (2004). Complete type III secretion system of a mesophilic Aeromonas hydrophila strain. Appl. Environ. Microbiol. 70, 6914-6919. doi: 10.1128/AEM.70.11.6914-6919.2004

Vogelaar, N. J., Jing, X., Robinson, H. H., and Schubot, F. D. (2010). Analysis of the crystal structure of the ExsC. ExsE complex reveals distinctive binding interactions of the Pseudomonas aeruginosa type III secretion chaperone ExsC with ExsE and ExsD. Biochemistry 49, 5870-5879. doi: 10.1021/bi100432e

Wilhelms, M., Gonzalez, V., Tomas, J. M., and Merino, S. (2013). Aeromonas hydrophila lateral flagellar gene transcriptional hierarchy. J. Bacteriol. 195, 1436-1445. doi: 10.1128/JB.01994-12

Yahr, T. L., and Frank, D. W. (1994). Transcriptional organization of the trans-regulatory locus which controls exoenzyme S synthesis in Pseudomonas aeruginosa. J. Bacteriol. 176, 3832-3838.

Yahr, T. L., Hovey, A. K., Kulich, S. M., and Frank, D. W. (1995). Transcriptional analysis of the Pseudomonas aeruginosa exoenzyme S structural gene. J. Bacteriol. 177, 1169-1178.

Yu, H. B., Kaur, R., Lim, S., Wang, X. H., and Leung, K. Y. (2007). Characterization of extracellular proteins produced by Aeromonas hydrophila AH-1. Proteomics 7, 436-449. doi: 10.1002/pmic.200600396

Zheng, Z., Chen, G., Joshi, S., Brutinel, E. D., Yahr, T. L., and Chen, L. (2007). Biochemical characterization of a regulatory cascade controlling transcription of the Pseudomonas aeruginosa type III secretion system. J. Biol. Chem. 282, 6136-6142. doi: 10.1074/jbc.M611664200

Conflict of Interest Statement: The authors declare that the research was conducted in the absence of any commercial or financial relationships that could be construed as a potential conflict of interest.

Copyright $\odot 2016$ Zhao and Shaw. This is an open-access article distributed under the terms of the Creative Commons Attribution License (CC BY). The use, distribution or reproduction in other forums is permitted, provided the original author(s) or licensor are credited and that the original publication in this journal is cited, in accordance with accepted academic practice. No use, distribution or reproduction is permitted which does not comply with these terms. 